\title{
Damping Performance of Dynamic Vibration Absorber in Nonlinear Simple Beam with 1:3 Internal Resonance
}

\author{
Yi-Ren Wang \\ Department of Aerospace Engineering, Tamkang University, NewTaipei City, Tamsui Dist., 25137, Taiwan.
}

\author{
Hsueh-Ghi Lu \\ RD Center-Environmental Reliability QA Div., Enterprise Products Testing Department, Pegatron Corporation, \\ Taipei City 11261, Taiwan.
}

\begin{abstract}
(Received 20 October 2014; accepted 17 November 2016)
This study investigated the damping effects of a dynamic vibration absorber (DVA) attached to a hinged-hinged nonlinear Euler-Bernoulli beam. The model constructed in this study was used to simulate suspended nonlinear elastic beam systems or vibrating elastic beam systems on a nonlinear Winkler-type foundation. This makes the modeling in this study applicable to suspension bridges, railway tracks, and even carbon nanotubes. The hinged-hinged beam in this study includes nonlinear stretching effects, which is why we adopted the method of multiple scales (MOMS) to analyze the frequency responses of fixed points in various modes. The use of amplitudes and vibration modes made it possible to examine the internal resonance. Our results indicate that particular elastic foundations or suspension systems can cause 1:3 internal resonance in a beam. The use of 3D maximum amplitude contour plots (3DMACPs) enabled us to obtain a comprehensive understanding of various DVA parameters, including mass, spring coefficient, and the location of DVA on the beam, and thereby combine them for optimal effect. Our results were verified using numerical calculations.
\end{abstract}

\section{INTRODUCTION}

Engineering from the scale of small mechanical components to that of aircraft wings and bridges must take into account the effects of vibration, which can cause fatigue, loosening of parts, and failure. Vibration can be linear or nonlinear, with the latter involving internal resonance that is complex and difficult to analyze. As described by Nayfeh and Mook, ${ }^{1}$ in the event that the natural vibration frequencies of various degrees of freedom (DOFs) are multiples of one another, excitation at high frequencies can increase amplitudes at low frequencies. This study sought to account for this unique nonlinear phenomenon. Nayfeh and Balachandran ${ }^{2}$ defined several stable conditions in nonlinear systems and presented a number of methods that could be used to determine system stability, and are therefore highly valuable as a reference in the analysis of system stability. Nayfeh and $\mathrm{Pai}^{3}$ investigated vibrations in nonlinear Euler-Bernoulli beams. According to Newton's laws, Euler's angle transformation, and the Karman-type strain-displacement relationship in order to derive 3D and 2D equations associated with nonlinear beams. These theoretical models have greatly benefited related research. Nayfeh and Mook $^{1}$ also proposed a number of methods with which to solve nonlinear systems, including the Poincaré method, the Lindstedt method, the average method, and MOMS, which is highly conducive to the analysis of damped vibration systems. $\mathrm{Ji}$ and $\mathrm{Zu}^{4}$ studied the rotating shaft system of a Timoshenko beam using MOMS to analyze the natural frequency responses of nonlinear systems. Nayfeh and Nayfeh ${ }^{5}$ employed MOMS to identify nonlinear modes and nonlinear frequencies, where- upon they applied the Galerkin method to the analysis of dynamic responses in a nonlinear beam. Analysis of vibrations in an elastic beam involves many factors. Van Horssen and Boertjens ${ }^{6}$ considered a suspension bridge under the influence of nonlinear aerodynamic effects using the theoretical model of a Euler-Bernoulli beam with linear springs to simulate vibrations in the bridge. They discovered that energy can shift between the first and third modes and that this is typical of nonlinear internal resonance. Their study prompted a great deal of research based on theoretical models. Theories similar to this, in which a beam is placed on an elastic foundation, have been widely applied to civil, mechanical, and aerospace engineering, by researchers such as Mundrey. ${ }^{7} \mathrm{Fu}$ et al. ${ }^{8}$ reported nonlinear vibrations in embedded carbon nanotubes (CNTs), based on research involving the use of nonlinear 2D EulerBernoulli beams to simulate vibrations in CNTs on elastic matrices. Shen ${ }^{9}$ used the model of a nonlinear 2D Euler-Bernoulli beam to analyze vibrations in a post-buckling beam placed on a double-layered elastic foundation, thereby demonstrating that the stiffness of elastic foundations has a significant influence on the vibration behavior of nonlinear beams.

Wang and $\mathrm{Chen}^{10}$ developed a damping approach to vibration reduction without the need to change the damper, but merely enable its relocation by having a mass-spring-damper vibration absorber hung from the optimal damping location on a rotating mechanism (a CD-ROM drive or the coupling system of rotary wings and swashplates). Wang and Chang ${ }^{11}$ studied nonlinear vibrations in a rigid plate using cubic springs to simulate supports at the four corners with two singlemass dampers hung from beneath the plate. The locations 
of the dampers were adjusted to achieve optimal damping effects. Dynamic vibration absorber (DVA) is a practical passive damping device for vibrating systems. For a linear vibrating beam, DVA can be pre-tuned to the modal frequency of the vibrating structure or the disturbance frequency to damp out the beam vibration. Wang and Chang ${ }^{12}$ discussed a hingedfree linear Euler-Bernoulli beam placed on a nonlinear elastic foundation. They found that placing a DVA of appropriate mass could prevent internal resonance and suppress vibrations in the beam. Nambu et al. ${ }^{13}$ studied the smart self-sensing active DVA attached on a flexible beams supported string. A small cantilevered piezoelectric transducer was used to realize this active DVA. They showed improvement control robustness upon passive DVAs. Tso et al. ${ }^{14}$ proposed a novel design method of hybrid dynamic vibration absorber (HVA) to suppress broadband vibration of the whole structure instead of just one point of the structure. Samani and Pellicano ${ }^{15}$ identified the optimal locations of a DVA applied to a simple beam subjected to regularly spaced and concentrated moving loads. The nonlinear stiffness of a nonlinear DVA possesses complex dynamic properties such as quasi-periodic, chaotic, and sub-harmonic responses. They discovered that the damping effects of a nonlinear non-symmetric dissipation DVA are superior to those of a linear DVA. In contrast, Wang and $\mathrm{Lin}^{16}$ used internal resonance contour plots (IRCPs) and flutter speed contour plots (FSCPs) to analyze nonlinear dynamic stability. Their results demonstrated that changing the location of the damper can prevent internal resonance and inhibit vibrations in the main body. Wang and Kuo ${ }^{17}$ examined the vibrations of a hinged-free nonlinear beam placed on a nonlinear elastic foundation. The 1:3 internal resonance in the first and second modes of the beam was found. An optimal location of an attached DVA was proposed to prevent internal resonance and suppress vibrations. Wang and $\mathrm{Tu}^{18}$ investigated the damping effects of a tuned mass damper (TMD) on a fixed-free 3D nonlinear beam resting on a nonlinear elastic foundation with existing internal resonances. They proposed that the locations of the maximum amplitudes between node points in the mode shapes and near the free end of the beam display the best damping effect for the fixed-free 3D beam. In the works of Wang and $\mathrm{Kuo}^{17}$ and Wang and $\mathrm{Tu},{ }^{18}$ the nonlinear geometry and nonlinear inertia were included in these two nonlinear beams. The conditions to trig the internal resonance were also investigated in their researches. The various beam boundary conditions were examined for better understanding the DVA damping effects on beam vibrations. Wang and Liang ${ }^{19}$ investigated the damping effects of vibration absorbers with a lumped mass on a hinged-hinged beam. This kind of vibration absorber is able to mitigate vibrations in mechanical or civil engineering structures on an elastic foundation; however, they are not applicable to all suspension systems. It is this shortcoming that we sought to address in this research.

Further consideration of resonance analysis of structures using the analytical and numerical strategies can be found in the work of Ansari et al. ${ }^{20}$ They derived a geometrically nonlinear beam model to simulate the nanobeam vibration under thermal environment. The material properties of nanobeams vary through the thickness direction with functionally graded (FG) distribution. The method of multiple scales was applied to de- termine the frequency response of the temperature and material properties. A 2-DOF nonlinear system (the main body and the controller (absorber)) with quadratic and cubic nonlinearities under external and parametric bounded excitations was considered in the work of Sayed and Kamel. ${ }^{21}$ The method of multiple scales was applied to determine the amplitudes and phases in the existence of internal resonance. The stability of the controlled system was studied. Ghayesh et al. ${ }^{22}$ investigated the axially moving beam with a three-to-one internal resonance. The coupled longitudinal and transverse displacements of the beam were studied numerically. The frequency response of the nonlinear forced system was solved by the pseudo-arc length continuation method and direct time integration. Ghayesh and Amabili ${ }^{23}$ provided a detailed discussion of an axially moving Timoshenko beam with an internal resonance. They used the Galerkin's scheme to discretize the nonlinear partial differential equations into a set of nonlinear ordinary differential equations. The pseudo-arc length continuation method was also employed to find the frequency response of the nonlinear forced system. The dynamic and resonant response were examined by the Poincaré maps. Stability properties with and without internal resonance were presented. Ansari et al. ${ }^{24}$ investigated the forced vibration of nonlinear magneto-electro-thermo elastic (METE) nanobeams. The Galerkin technique was applied to obtain a time-varying set of ordinary differential equations. The pseudo-arc length continuum scheme was also used to solve this nonlinear beam system. Ansari et al. ${ }^{25}$ applied the similar numerical strategies in the analysis of the forced vibration behavior of reinforced single-walled carbon nanotubes (SWCNTs). Different effects of SWCNT parameters were examined by the frequency responses. Their results showed that the amplitude peak reduces when the nanotube volume fraction or dimensionless damping parameter gets larger. Ansari et al. ${ }^{26}$ also invested the surface stress effect on the vibrations of nanobeams. The generalized differential quadrature method and the shifted ChebyshevGauss-Lobatto grid points were employed to solve the nonlinear problem. The frequency response of nanobeams including the effect of surface stress was investigated. Ansari et al. ${ }^{27}$ performed a numerical study of two-phase $\mathrm{BiTiO}_{3}-\mathrm{CoFe}_{2} \mathrm{O}_{4}$ composites nanobeams subjected to various boundary conditions. The Galerkin and pseudo arc-length methods were employed for solving nonlinear problems. They also provided a novel technique of periodic time differential operators for the time domain discretization. Ansari and Gholami ${ }^{28}$ further investigated the surface stress and surface inertia effects on the rectangular nanoplates. The equations of motion were solved numerically for the rectangular Si and Al nanoplates with various boundary supports by means of the generalized differential quadrature method. The hardening nonlinearity and surface effects showed considerable influences on nanoplates.

This study considered a nonlinear hinged-hinged EulerBernoulli beam supported or suspended using nonlinear springs, including internal resonance and the coupling of multiple modes. This system is able to simulate a vibrating mechanical system suspended or placed on an elastic medium, such as a suspension bridge or the tracks of subways or highspeed trains. This system can also be used to simulate CNTs placed on an elastic matrix. The main body in this study was 


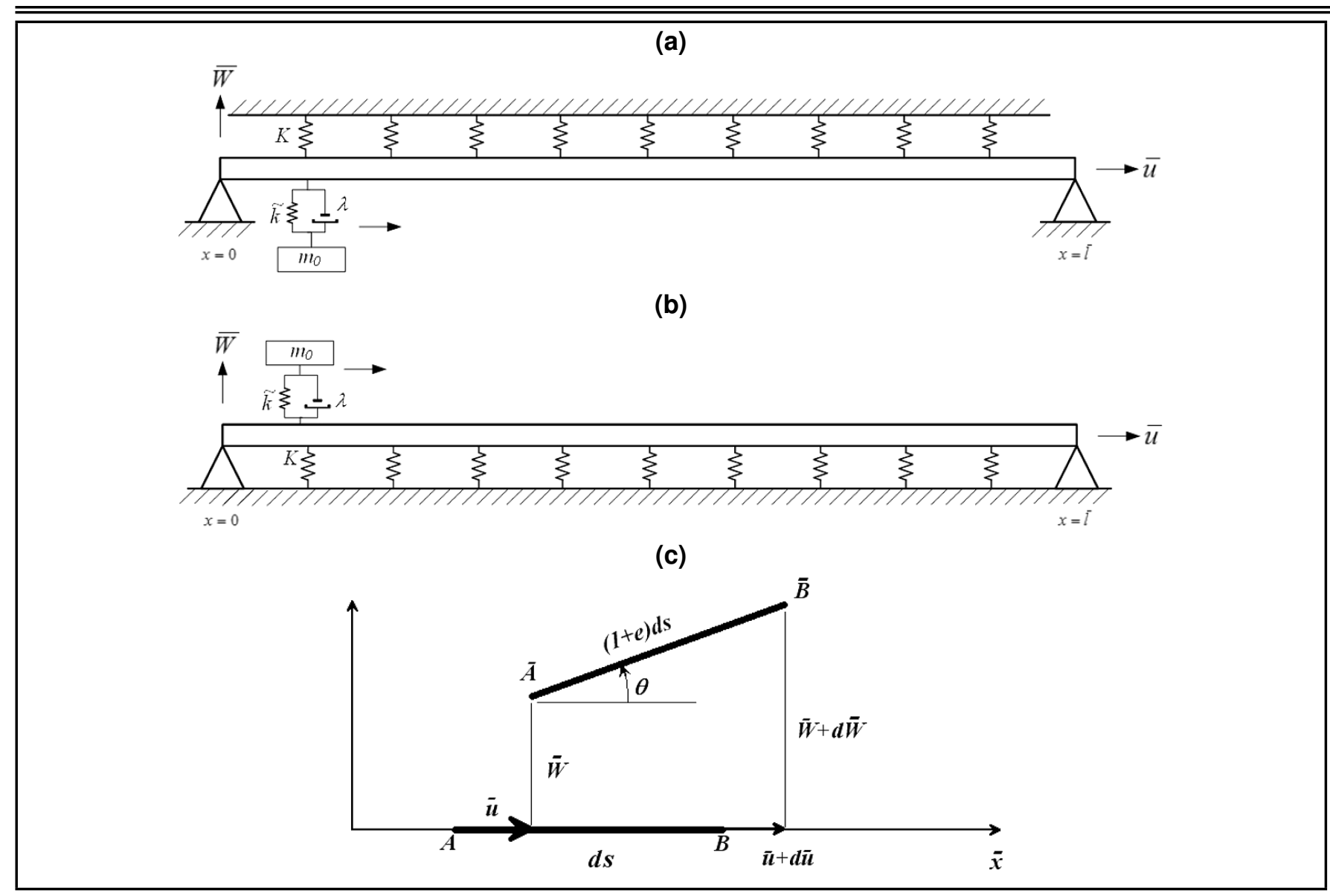

Figure 1. A schematic of the hinged-hinged beam system, (a) suspension beam, (b) Winkler type foundation, (c) relationship between beam displacement and rotation angle.

subjected to a harmonic force. We used a mass-spring DVA to reduce vibrations, taking into account the effects of stretching. The influence of parameters, such as the mass, elasticity coefficient, and location of the DVA on damping performance were analyzed extensively. We used 3D maximum amplitude contour plots (3DMACPs) to identify the optimal combination of parameters as well as numerical calculations to verify the accuracy of the results.

\section{DEVELOPMENT OF THE THEORETICAL MODEL}

The main body in this study comprised of a nonlinear elastic beam supported or suspended by nonlinear springs to simulate an elastic foundation or suspension cables. Figure 1 illustrates the coordinate system, where $m$ denotes the mass of the elastic beam per unit length; $A, E$, and $I_{A}$ are the cross-section area, Young's modulus, and moment of inertia of the beam, respectively; $k$ is the liner spring constant of the elastic foundation; $\beta$ signifies the nonlinear spring constant; $\bar{\mu}$ is the linear damping coefficient of the beam; and $\bar{f}_{s}$ and $\bar{g}_{s}$ denote the linear spring constant and the damping coefficient of the DVA, respectively.

\subsection{Deduction and Non-Dimensionalization of Nonlinear Equations of Motion}

Figures 1a and $1 \mathrm{~b}$ exhibit the hinged-hinged nonlinear beam employed in this study. We referred to the nonlinear beam theory proposed by Nayfeh and Pai ${ }^{9}$ and used Newtonian's Laws to develop a complete model including the elastic foundation and the DVA. We first considered the initial status of the beam when it is straight and assumed that each cross-section is a plane that follows the stress-strain laws. According to Nayfeh and $\mathrm{Pai}^{9}$ and from Fig. 1c, the axial strain $e$ and the rotation angle $\theta$ are related to $\bar{u}^{\prime}$ and $\bar{W}^{\prime}$ as $e=\sqrt{\left(1+\bar{u}^{\prime}\right)^{2}+\bar{W}^{\prime 2}}-1$, $\cos \theta=\left(1+\bar{u}^{\prime}\right) /(1+e)$, and $\sin \theta=\bar{W}^{\prime} /(1+e)$. Assuming $\bar{u}$ and $\bar{W}$ are small but finite, then $e$ and $\theta$ can be further expanded as $e=\bar{u}^{\prime}+\bar{W}^{\prime 2} / 2-\left(\bar{u}^{\prime} \bar{W}^{2}\right) / 2 \ldots$ and $\theta=\tan ^{-1}\left(\bar{W}^{\prime} /\left(1+\bar{u}^{\prime}\right)\right)=\bar{W}^{\prime}-\bar{u}^{\prime} \bar{W}^{\prime}+\bar{u}^{\prime 2} \bar{W}^{\prime}+\bar{u}^{\prime 2} \bar{W}^{\prime}-$ $\bar{W}^{\prime 3} / 3 \ldots$ Using Newton's Laws, Euler's angle transformation, and Taylor series expansion, we obtained the basic equations of motion for the nonlinear beam. We excluded any rotations in the beam, limiting it to planar motions. Thus, the equations of motion for the 2D beam are as follows:

$$
\begin{gathered}
m \ddot{\bar{u}}-E A \bar{u}^{\prime \prime}=E A\left(\frac{1}{2} \bar{W}^{2}-\bar{u}^{\prime} \bar{W}^{\prime 2}\right)^{\prime}+ \\
E I_{A}\left[\bar{W}^{\prime}\left(\bar{W}^{\prime \prime \prime}-\bar{u}^{\prime \prime \prime} \bar{W}^{\prime}-2 \bar{u}^{\prime \prime} \bar{W}^{\prime \prime}-3 \bar{u}^{\prime} \bar{W}^{\prime \prime \prime}\right)\right]^{\prime} ; \\
m \ddot{\bar{W}}-E I_{A} \bar{W}^{i v}=E A\left(\bar{u}^{\prime} \bar{W}^{\prime}-\bar{u}^{\prime 2} \bar{W}^{\prime}+\frac{1}{2} \bar{W}^{\prime 3}\right)^{\prime}+ \\
E I_{A}\left[\bar{u}^{\prime} \bar{W}^{\prime \prime \prime}+\left(\bar{u}^{\prime} \bar{W}^{\prime}\right)^{\prime \prime}-\left(\bar{u}^{\prime 2}-\bar{W}^{2}\right) \bar{W}^{\prime \prime \prime}-\right. \\
\left.\bar{u}^{\prime}\left(\bar{u}^{\prime} \bar{W}^{\prime}\right)^{\prime \prime}-\left(\bar{u}^{\prime 2} \bar{W}^{\prime}-\frac{1}{3} \bar{W}^{\prime 3}\right)^{\prime \prime}\right]^{\prime}
\end{gathered}
$$


where ()$^{\prime}$ and $\left({ }^{\circ}\right)$ denote $d / d \bar{x}$ and $d / d \bar{t}$, respectively. The $\bar{u}$ and $\bar{W}$ represent the beam displacement in the longitudinal direction ( $x$-axis) and transversal direction (vertical-axis), respectively. The system considered in this study is a slender elastic beam; therefore, the longitudinal inertia force $m \ddot{\bar{u}}$ in Eq. (1) can be disregarded. The beam is hinged at both ends, and no longitudinal forces were imposed. This made it necessary to consider the effects of stretching. The boundary conditions are as follows:

$$
\begin{array}{lll}
\bar{u}(0, \bar{t})=0 ; & \bar{u}(\bar{l}, \bar{t})=0 ; & \bar{W}(0, \bar{t})=0 \\
\bar{W}(\bar{l}, \bar{t})=0 ; & \bar{W}^{\prime \prime}(0, \bar{t})=0 ; & \bar{W}^{\prime \prime}(\bar{l}, \bar{t})=0 .
\end{array}
$$

From Eq. (1), we can express the $\bar{u}^{\prime \prime}$ as

$$
\bar{u}^{\prime \prime}=-\left(\frac{1}{2} \bar{W}^{2}\right)^{\prime}+\ldots .
$$

The integral of Eq. (4) is

$$
\begin{aligned}
& \bar{u}^{\prime}=-\frac{1}{2} \bar{W}^{2}+c_{1}(\bar{t}) \\
& \bar{u}=-\frac{1}{2} \int_{0}^{\bar{l}} \bar{W}^{\prime 2} d \bar{x}+c_{1}(\bar{t}) \bar{x}+c_{2}(\bar{t}) .
\end{aligned}
$$

Substituting Eq. (5) into the boundary conditions in Eq. (3) produces

$$
c_{2}(\bar{t})=0 ; \quad c_{1}(\bar{t})=\frac{1}{2 \bar{l}} \int_{0}^{\bar{l}} \bar{W}^{\prime 2} d \bar{x} .
$$

After substituting Eqs. (5) and (6) into Eq. (2), we can simplify the equations of motion to an equation in which vibrations are presented in the $\bar{W}$ direction. We included the structural damping term $\bar{\mu} \dot{\bar{W}}$ of the elastic beam, the linear and nonlinear elastic terms $k\left[\bar{W}+\beta \bar{W}^{3}\right]$ of the support or suspension springs, and the uniform distributed load $\bar{F}=\bar{F} e^{i \bar{\Omega} \bar{t}}$, where $\bar{\mu}$ is the structural damping coefficient; $k$ and $\beta$ represent the linear and nonlinear elastic constant of the support or suspension springs, respectively. The hanging DVA was regarded an external force $\left\{\bar{f}_{s}\left[\bar{W}(x, t)-\bar{W}_{D}\right]+\bar{g}_{s}\left[\dot{\bar{W}}(x, t)-\dot{W}_{D}\right]\right\} \delta\left[x-\bar{l}_{D}\right]$, where $\bar{f}_{s}$ and $\bar{g}_{s}$ denote the spring constant and the damping coefficient of the DVA, respectively. It is also noted that $\bar{l}$ represents the beam length and the $\bar{W}_{D}$ and $\bar{l}_{D}$ represent the displacement and the location of the DVA, respectively. We used Newton's laws to deduce the equation of motion before reorganizing and nondimensionalizing it, thereby obtaining the following:

$$
\begin{gathered}
\stackrel{* *}{W}+W^{i v}+\bar{\omega}^{2} W+\mu \stackrel{*}{W}+K W^{3}+\left\{\widetilde{k}\left[W(x, \tau)-W_{D}\right]+\right. \\
\left.\lambda\left[\stackrel{*}{W}(x, \tau)-\stackrel{*}{W}_{D}\right]\right\} \delta\left[x-l_{D}\right]= \\
\frac{1}{2} \hat{A}\left[\int_{0}^{l} W^{\prime 2} d x\right] W^{\prime \prime}+F e^{i \Omega \tau} .
\end{gathered}
$$

The boundary conditions in Eq. (3) are thus

$$
\begin{array}{ll}
W(0, \tau)=0 ; & W^{\prime \prime}(0, \tau)=0 \\
W(l, \tau)=0 ; & W^{\prime \prime}(l, \tau)=0
\end{array}
$$

The non-dimensionalized equation of motion of the DVA is

$$
m_{0} \stackrel{* *}{W}_{D}(\tau)-[\widetilde{k}(u)+\lambda(\stackrel{*}{u})]=0
$$

where

$$
u(\tau)=W\left(l_{D}, \tau\right)-W_{D}(\tau) .
$$

Equation (7) is the non-dimensionalized equation of motion of the main body, in which ()$^{\prime}$ and $\left(^{*}\right)$ represent $d / d x$ and $d / d \tau$; $\bar{\omega}^{2}$ is the ratio between the linear elasticity coefficient of the support or suspension springs and the elasticity coefficient of the elastic beam; $\mu$ is the non-dimensionalized beam damping coefficient; $K$ is the ratio between the nonlinear vibration frequency of the supports or suspension springs and the vibration frequency of the elastic beam, $l_{D}$ is the non-dimensionalized DVA position, $\hat{A}$ is the non-dimensional beam rigidity; and $\widetilde{k}$ and $\lambda$ are the non-dimensionalized spring constant and damping coefficient of the DVA, respectively. Appendix 1 contains the definitions of the other non-dimensionalized coefficients.

\subsection{Application of MOMS}

This study adopted MOMS to analyze the frequency response and fixed points of the nonlinear equation, which involves dividing the time scale into fast and slow time scales. Suppose $T_{0}$ is the fast-time term, $T_{1}, T_{2}, \ldots$ are the slow-time terms, and $W(x, \tau, \varepsilon)=W_{0}\left(x, t_{0}, t_{1}, \ldots\right)+$ $\varepsilon W_{1}\left(x, t_{0}, t_{1}, \ldots\right)$, where $\varepsilon$ is the time scale of small disturbances and is an infinitesimal value. We also considered the damping and nonlinear terms as well as the external force as small disturbance terms and set the order at $\varepsilon^{1}$ to facilitate analysis. We substituted these principles into Eq. (7) and disregarded the influence of high-order terms such as $\varepsilon^{2}, \varepsilon^{3}, \ldots$ on the system. The terms of $\varepsilon^{0}$ are as follows:

$$
\frac{\partial^{2} W_{0}}{\partial T_{0}^{2}}+\frac{\partial^{4} W_{0}}{\partial x^{4}}+\bar{\omega}^{2} W_{0}=0 .
$$

The terms of $\varepsilon^{1}$ are as follows:

$$
\begin{gathered}
\frac{\partial^{2} W_{1}}{\partial T_{0}^{2}}+\frac{\partial^{4} W_{1}}{\partial x^{4}}+\bar{\omega}^{2} W_{1}=\frac{\partial^{2} W_{0}}{\partial x^{2}} \hat{A}\left[\frac{1}{2} \int_{0}^{1}\left(\frac{\partial W_{0}}{\partial x}\right)^{2} d x\right]+ \\
F-[\widetilde{k}(u)+\lambda(\stackrel{*}{u})] \delta\left[x-l_{D}\right]-2 \frac{\partial^{2} W_{0}}{\partial T_{0} \partial T_{1}}- \\
\mu \frac{\partial W_{0}}{\partial T_{0}}-K W_{0}^{3} .
\end{gathered}
$$

The boundary conditions corresponding to $\varepsilon^{0}$ and $\varepsilon^{1}$ equations are as follows: $W(0, \tau)=0, W^{\prime \prime}(0, \tau)=0, W(l, \tau)=0$, $W^{\prime \prime}(l, \tau)=0$. Considering that the DVA is linear, MOMS need not be used to divide the time scale, such that only $\varepsilon^{0}$ is considered in the DVA equation of motion.

\section{ANALYSIS OF INTERNAL RESONANCE CONDITIONS}

\subsection{Equation of Motion for the Nonlinear Beam Without DVA}

The process of modeling the system without the DVA is the same as the development of the theoretical model in Section 2. Thus, we only provide a brief description below. 
After dropping the DVA from the equation of motion in the previous section, we obtain the following:

$$
\begin{aligned}
& \stackrel{* *}{W}+W^{i v}+\bar{\omega}^{2} W= \\
& \quad \frac{1}{2} \hat{A}\left(\int_{0}^{l} W^{\prime 2} d x\right) W^{\prime \prime}-\mu W^{*}-K W^{3}+F e^{i \Omega \tau}
\end{aligned}
$$

In this model, the two ends are hinged; therefore, the boundary conditions are

$$
\begin{array}{ll}
W(0, \tau)=0 ; & W^{\prime \prime}(0, \tau)=0 \\
W(l, \tau)=0 ; & W^{\prime \prime}(l, \tau)=0
\end{array}
$$

Note that in this non-dimensionalized circumstance, $l=1$.

\subsection{Conditions of Internal Resonance in the Beam System without DVA}

We applied MOMS and the terms comprising $\varepsilon^{0}$ are as follows:

$$
\frac{\partial^{2} W_{0}}{\partial T_{0}^{2}}+\frac{\partial^{4} W_{0}}{\partial x^{4}}+\bar{\omega}^{2} W_{0}=0
$$

Terms comprising $\varepsilon^{1}$ are as follows:

$$
\begin{gathered}
\frac{\partial^{2} W_{1}}{\partial T_{0}^{2}}+\frac{\partial^{4} W_{1}}{\partial x^{4}}+\bar{\omega}^{2} W_{1}=\frac{1}{2} \hat{A} \frac{\partial^{2} W_{0}}{\partial x^{2}}\left[\int_{0}^{1}\left(\frac{\partial W_{0}}{\partial x}\right)^{2} d x\right]+ \\
F-2 \frac{\partial^{2} W_{0}}{\partial T_{0} \partial T_{1}}-\mu \frac{\partial W_{0}}{\partial T_{0}}-K W_{0}^{3}
\end{gathered}
$$

Suppose that the general solution to $W(x, \tau)$ is

$$
W(x, \tau)=\left(A\left(T_{1}\right) e^{-i \zeta} e^{i \omega T_{0}}+\bar{A}\left(T_{1}\right) e^{i \zeta} e^{-i \omega T_{0}}\right) \phi(x)
$$

where $\zeta$ is the phase angle. Also, let

$$
\phi(x)=E_{1} \sin \gamma x+E_{2} \cos \gamma x+E_{3} \sinh \gamma x+E_{4} \cosh \gamma x .
$$

By substituting the equation above into the boundary conditions, we obtain the eigenvalues $\gamma_{n}=n \pi / l, n=1,2,3, \ldots$, and $l=1$, whereas the mode shape $\phi_{n}(x)$ can be expressed as $\phi_{n}(x)=\sin \gamma_{n} x$. After using orthogonal method, $W_{0 n}(x, \tau)$ can be written as

$$
\begin{aligned}
& W_{0 n}(x, \tau)= \\
& \sum_{n=1}^{\infty}\left(B_{n}\left(T_{1}\right) e^{-i \zeta_{n}} e^{i \omega_{n} T_{0}}+\bar{B}_{n}\left(T_{1}\right) e^{i \zeta_{n}} e^{-i \omega_{n} T_{0}}\right) \phi_{n}(x)= \\
& \sum_{n=1}^{\infty} \xi_{0 n}(\tau) \phi_{n}(x)
\end{aligned}
$$

Using the orthogonal method, we multiply Eq. (15) by $\phi_{n}(x)$ and integrate it from 0 to $l(l=1$ (normalized)) and obtain

$$
\begin{gathered}
\xi_{0 m}^{* *}(\tau)+\left(\gamma_{m}^{4}+\bar{\omega}^{2}\right) \xi_{0 m}(\tau)=0 \\
\xi_{1 m}^{* *}(\tau)+\left(\gamma_{m}^{4}+\bar{\omega}^{2}\right) \xi_{1 m}(\tau)= \\
-\frac{1}{2} \gamma_{m}^{2} \xi_{0 m} \hat{A}\left[\int_{0}^{1}\left(\sum_{p=1}^{\infty} \gamma_{p}^{2} \xi_{0 p}^{2} \cos ^{2} \gamma_{p} x\right) d x\right]+ \\
\bar{F} \frac{\int_{0}^{1} \phi_{m} d x}{\int_{0}^{1} \phi_{m}^{2} d x}-2 \frac{\partial^{2} \xi_{0 m}(\tau)}{\partial T_{0} \partial T_{1}}-\mu \frac{\partial \xi_{0 m}(\tau)}{\partial T_{0}}- \\
\frac{K}{\int_{0}^{1} \phi_{m}^{2} d x} \int_{0}^{1}\left(\sum_{i}^{\infty} \xi_{0 i} \phi_{i}\right)\left(\sum_{j}^{\infty} \xi_{0 j} \phi_{j}\right)\left(\sum_{k}^{\infty} \xi_{0 k} \phi_{k}\right) \phi_{m} d x
\end{gathered}
$$

and set the natural frequencies of the various modes in the system as $\omega_{n}=\sqrt{\gamma_{m}^{4}+\bar{\omega}^{2}}$. To determine the existence of internal resonance in the beam system, we must derive the $\bar{\omega}^{2}$ conditions capable of causing internal resonance. Among these conditions, Eq. (21) contains $\xi_{0 i} \xi_{0 j} \xi_{0 k}$, an infinite series of nonlinear terms. This hinders the determination of internal resonance with $\bar{\omega}^{2}$; therefore, we used the method proposed by Van Horssen ${ }^{6}$ and followed the procedure described by Wang and Liang ${ }^{19}$ to derive the internal resonance conditions created by $\bar{\omega}^{2}$. We first observe the integration term $\int_{0}^{1} \phi_{i}(x) \phi_{j}(x) \phi_{k}(x) \phi_{m}(x) d x$ on the right side of Eq. (21), which is the orthogonal product of $\phi_{m}(x)$ and $\phi_{i}(x) \phi_{j}(x) \phi_{k}(x)$. Based on the orthogonal method, the integration term in Eq. (21) may contribute to the secular terms if it does not equal 0 and $m= \pm i \pm j \pm k$. Furthermore, as the dominant term in $\phi_{m}(x)$ is $\sin \gamma_{m} x$, we can only observe whether $\pm \gamma_{i} \pm \gamma_{j} \pm \gamma_{k}$ equals $\gamma_{m}$ in the product terms in $\int_{0}^{1} \phi_{i}(x) \phi_{j}(x) \phi_{k}(x) \phi_{m}(x) d x$ in order to confirm the existence of orthogonality before addressing the question of whether the harmonic frequencies extending from the harmonic combination are secular terms. As $\gamma_{m}=n \pi>0$, we can eliminate $-\gamma_{m}=\gamma_{i}+\gamma_{j}-\gamma_{k}$ and $-\gamma_{m}=\gamma_{i}-\gamma_{j}+\gamma_{k}$ from the possibilities in $\pm \gamma_{m}= \pm \gamma_{i} \pm \gamma_{j} \pm \gamma_{k}$. Since $\gamma_{m}=$ $\gamma_{i}+\gamma_{j}-\gamma_{k},-\gamma_{m}=\gamma_{i}-\gamma_{j}-\gamma_{k}$, and $\gamma_{m}=\gamma_{i}-\gamma_{j}+\gamma_{k}$ are similar; therefore, we need only to discuss three combinations: $\gamma_{m}=\gamma_{i}+\gamma_{j}+\gamma_{k}, \gamma_{m}=\gamma_{i}-\gamma_{j}-\gamma_{k}$, and $\gamma_{m}=\gamma_{i}+\gamma_{j}-\gamma_{k}$ from the possibilities in $\pm \gamma_{m}= \pm \gamma_{i} \pm \gamma_{j} \pm \gamma_{k}$. Furthermore, in the product $\xi_{0 i} \xi_{0 j} \xi_{0 k}$ on the right side of Eq. (21), there exists secular terms that are equal to the harmonics on the left side, which prevents the system from reaching convergence. Of these terms, only those that exist in the form of $e^{i\left( \pm \omega_{i} \pm \omega_{j} \pm \omega_{k}\right) T_{0}}$ are possible. Hence, we only considered the combinations in $\omega_{m}= \pm \omega_{i} \pm \omega_{j} \pm \omega_{k}$, which is why we discussed the following combinations according to conditions described above:
(A) $\left\{\begin{array}{l}\gamma_{m}=\gamma_{i}+\gamma_{j}+\gamma_{k} \\ \left(\gamma_{m}^{4}+\bar{\omega}^{2}\right)^{\frac{1}{2}}= \pm\left(\gamma_{i}^{4}+\bar{\omega}^{2}\right)^{\frac{1}{2}} \pm\left(\gamma_{j}^{4}+\bar{\omega}^{2}\right)^{\frac{1}{2}} \pm\left(\gamma_{k}^{4}+\bar{\omega}^{2}\right)^{\frac{1}{2}}\end{array} ;\right.$
(B) $\left\{\begin{array}{l}\gamma_{m}=-\gamma_{i}-\gamma_{j}+\gamma_{k} \\ \left(\gamma_{m}^{4}+\bar{\omega}^{2}\right)^{\frac{1}{2}}= \pm\left(\gamma_{i}^{4}+\bar{\omega}^{2}\right)^{\frac{1}{2}} \pm\left(\gamma_{j}^{4}+\bar{\omega}^{2}\right)^{\frac{1}{2}} \pm\left(\gamma_{k}^{4}+\bar{\omega}^{2}\right)^{\frac{1}{2}}\end{array} ;\right.$
(C) $\left\{\begin{array}{l}\gamma_{m}=\gamma_{i}+\gamma_{j}-\gamma_{k} \\ \left(\gamma_{m}^{4}+\bar{\omega}^{2}\right)^{\frac{1}{2}}= \pm\left(\gamma_{i}^{4}+\bar{\omega}^{2}\right)^{\frac{1}{2}} \pm\left(\gamma_{j}^{4}+\bar{\omega}^{2}\right)^{\frac{1}{2}} \pm\left(\gamma_{k}^{4}+\bar{\omega}^{2}\right)^{\frac{1}{2}}\end{array}\right.$ 
We determined the following ranges: when $0 \leq \bar{\omega}^{2} \leq 10 \pi^{4}$ and $\gamma_{i}, \gamma_{j}, \gamma_{k} \geq \pi$, solutions contributing to secular terms can be found in these combinations; thus, the discussion on the combinations and probabilities is as follows.

Case (A) $\gamma_{m}=\gamma_{i}+\gamma_{j}+\gamma_{k}$.

Combination (1): $\left(\gamma_{m}^{4}+\bar{\omega}^{2}\right)^{\frac{1}{2}}=\left(\gamma_{i}^{4}+\bar{\omega}^{2}\right)^{\frac{1}{2}}+\left(\gamma_{j}^{4}+\bar{\omega}^{2}\right)^{\frac{1}{2}}+$ $\left(\gamma_{k}^{4}+\bar{\omega}^{2}\right)^{\frac{1}{2}}$. Using inequality $h^{2}<\left(h^{4}+\bar{\omega}^{2}\right)^{\frac{1}{2}} \leq h^{2}-$ $a^{2}+\left(a^{4}+\bar{\omega}^{2}\right)^{\frac{1}{2}}$ and let $\gamma_{m}^{2}=\gamma_{i}^{2}+\gamma_{j}^{2}+\gamma_{k}^{2}+\lambda$, where $\lambda=2\left(\gamma_{i} \gamma_{j}+\gamma_{j} \gamma_{k}+\gamma_{i} \gamma_{k}\right)$. This enables us to derive the following inequality: $\gamma_{m}^{2}<\left(\gamma_{m}^{4}+\bar{\omega}^{2}\right)^{\frac{1}{2}} \leq \gamma_{i}^{2}+\gamma_{j}^{2}+\gamma_{k}^{2}-$ $3 \pi^{2}+3\left(\pi^{4}+\bar{\omega}^{2}\right)^{\frac{1}{2}}$, such that $2\left(\gamma_{i} \gamma_{j}+\gamma_{j} \gamma_{k}+\gamma_{i} \gamma_{k}\right)<-3 \pi^{2}+$ $3\left(\pi^{4}+\bar{\omega}^{2}\right)^{\frac{1}{2}}$. We also know that if $\gamma_{m}^{2}-\pi^{2}+\left(\pi^{4}+\bar{\omega}^{2}\right)^{\frac{1}{2}} \geq$ $\left(\gamma_{m}^{4}+\bar{\omega}^{2}\right)^{\frac{1}{2}}>\gamma_{i}^{2}+\gamma_{j}^{2}+\gamma_{k}^{2}$, we can get $\pi^{2}-\left(\pi^{4}+\bar{\omega}^{2}\right)^{\frac{1}{2}}<$ $\lambda<-3 \pi^{2}+3\left(\pi^{4}+\bar{\omega}^{2}\right)^{\frac{1}{2}}$ and $\gamma_{i}, \gamma_{j}, \gamma_{k} \geq \pi$, which means that $\lambda \geq 6 \pi^{2}$. If $\bar{\omega}^{2} \leq 8 \pi^{4}$, then the inequality cannot be satisfied. If $\gamma_{i}=\gamma_{j}=\gamma_{k}=\pi$, then $\gamma_{m}=3 \pi$ and $\left((3 \pi)^{4}+\bar{\omega}^{2}\right)^{\frac{1}{2}}=3\left(\pi^{4}+\bar{\omega}^{2}\right)^{\frac{1}{2}}$, whereby we have the solution $\bar{\omega}^{2}=9 \pi^{4}$.

Combination (2): $\left(\gamma_{m}^{4}+\bar{\omega}^{2}\right)^{\frac{1}{2}}=\left(\gamma_{i}^{4}+\bar{\omega}^{2}\right)^{\frac{1}{2}}+\left(\gamma_{j}^{4}+\bar{\omega}^{2}\right)^{\frac{1}{2}}-$ $\left(\gamma_{k}^{4}+\bar{\omega}^{2}\right)^{\frac{1}{2}}$. This combination has no solution. We can use the analysis method from Combination (1); however, the symmetric relationship means that equations with a negative sign have no solution.

Combination (3): $\left(\gamma_{m}^{4}+\bar{\omega}^{2}\right)^{\frac{1}{2}}=\left(\gamma_{i}^{4}+\bar{\omega}^{2}\right)^{\frac{1}{2}}-\left(\gamma_{j}^{4}+\bar{\omega}^{2}\right)^{\frac{1}{2}}-$ $\left(\gamma_{k}^{4}+\bar{\omega}^{2}\right)^{\frac{1}{2}}$. This combination has no solution. We can use the analysis method from Combination (1); however, the symmetric relationship means that equations with two negative signs have no solution.

Case (B) $\gamma_{m}=-\gamma_{i}-\gamma_{j}+\gamma_{k}$ is the same as Case (A), because $m$ and $k$ are symmetric.

Case (C) $\gamma_{m}=\gamma_{i}+\gamma_{j}-\gamma_{k}$ has solutions, but when $\bar{\omega}^{2}=$ $9 \pi^{4}$, internal resonance cannot occur within any of the modes with $m=1 \sim 4$.

Based on the above analysis, we can see that within the limited range of $\bar{\omega}^{2}<10 \pi^{4}, 1: 3$ internal resonance occurs only in modes $m=1$ and $m=3$ when $\bar{\omega}^{2}=9 \pi^{4}$. When $m=1$, $\omega_{1}=\sqrt{\gamma_{1}^{4}+\bar{\omega}^{2}}=\sqrt{\pi^{4}+9 \pi^{4}}=\sqrt{10} \pi^{2}$; when $m=3$, $\omega_{3}=\sqrt{\gamma_{3}^{4}+\bar{\omega}^{2}}=\sqrt{(3 \pi)^{4}+9 \pi^{4}}=3 \sqrt{10} \pi^{2}$, which means that $3 \omega_{1}=\omega_{3}$. We need only consider the internal resonance in the $1^{\text {st }}$ and $3^{\text {rd }}$ modes.

\subsection{Frequency Responses in Beam System without DVA}

To analyze the frequency responses of the system and derive the fixed point plots, we assume that the elastic beam is subject to a uniform distributed force:

$$
\begin{gathered}
\hat{f}_{m} e^{i r \tau}=\hat{f}_{m} e^{i\left(\omega_{m}+\varepsilon \sigma\right) T_{0}}=\hat{f}_{m}\left(e^{i \varepsilon \sigma T_{0}} e^{i \omega_{m} T_{0}}\right)= \\
\hat{f}_{m} e^{i \sigma T_{1}} e^{i \omega_{m} T_{0}} .
\end{gathered}
$$

With regard to the $1^{\text {st }}$ mode $(m=1)$, we must select the secular terms with harmonics $\omega_{1}$ and $\omega_{3}-2 \omega_{1}$. Thus, from the right side of Eq. (21), we select terms proportional to $e^{i \omega_{1} T_{0}}$ and $e^{i\left(\omega_{3}-2 \omega_{1}\right) T_{0}}$ (these are called secular terms):

$$
\begin{aligned}
- & \frac{1}{2} \hat{A}\left[\gamma_{1}^{4}\left(\int_{0}^{1} \cos ^{2} \gamma_{1} x d x\right)\left(3 B_{1} B_{1} \bar{B}_{1} e^{-i \zeta_{1}}\right)+\right. \\
& \left.\gamma_{1}^{2} \gamma_{3}^{2}\left(\int_{0}^{1} \cos ^{2} \gamma_{3} x d x\right)\left(2 B_{1} B_{3} \bar{B}_{3} e^{-i \zeta_{1}}\right)\right]+ \\
& A_{21} \bar{F}_{1}-2 i \omega_{1} B_{1}^{\prime}\left(T_{1}\right) e^{-i \zeta_{1}}-2 \omega_{1} \zeta_{1}^{\prime} B_{1}\left(T_{1}\right) e^{-i \zeta_{1}}- \\
& \mu i \omega_{1} B_{1}\left(T_{1}\right) e^{-i \zeta_{1}}-A_{31} K\left(6 B_{1} B_{3} \bar{B}_{3} \int_{0}^{1} \phi_{1}^{2} \phi_{3}^{2} d x e^{-i \zeta_{1}}+\right. \\
& \left.3 B_{1}^{2} \bar{B}_{1} \int_{0}^{1} \phi_{1}^{4} d x e^{-i \zeta_{1}}+3 \bar{B}_{1}^{2} B_{3} \int_{0}^{1} \phi_{1}^{3} \phi_{3} d x e^{i\left(2 \zeta_{1}-\zeta_{3}\right)}\right) .
\end{aligned}
$$

In terms of the $3^{\text {rd }}$ mode $(m=3)$, we must select the secular terms with harmonics $\omega_{3}$ and $3 \omega_{1}$. Thus, from the right side of Eq. (21) and let $m=3$, we select terms proportional to $e^{i \omega_{3} T_{0}}$ and $e^{i 3 \omega_{1} T_{0}}$ (these are called secular terms) as shown below:

$$
\begin{aligned}
& -\frac{1}{2} \hat{A}\left[\gamma_{3}^{4}\left(\int_{0}^{1} \cos ^{2} \gamma_{3} x d x\right)\left(3 B_{3} B_{3} \bar{B}_{3} e^{-i \zeta_{3}}\right)+\right. \\
& \left.\gamma_{1}^{2} \gamma_{3}^{2}\left(\int_{0}^{1} \cos ^{2} \gamma_{1} x d x\right)\left(2 B_{3} B_{1} \bar{B}_{1} e^{-i \zeta_{3}}\right)\right]+ \\
& A_{23} \bar{F}_{3}-2 i \omega_{3} B_{3}^{\prime}\left(T_{1}\right) e^{-i \zeta_{3}}-2 \omega_{3} \zeta_{3}^{\prime} B_{3}\left(T_{1}\right) e^{-i \zeta_{3}}- \\
& \quad \mu i \omega_{3} B_{3}\left(T_{1}\right) e^{-i \zeta_{3}}-A_{33} K\left(B_{1}^{3} \int_{0}^{1} \phi_{1}^{3} \phi_{3} d x e^{-i 3 \zeta_{1}}+\right. \\
& \left.6 B_{1} \bar{B}_{1} B_{3} \int_{0}^{1} \phi_{1}^{2} \phi_{3}^{2} d x e^{-i \zeta_{3}}+3 B_{3}^{2} \bar{B}_{3} \int_{0}^{1} \phi_{3}^{4} d x e^{-i \zeta_{3}}\right) .
\end{aligned}
$$

The selected secular terms are designated as being equal to 0 in order to derive a solvability condition. Below, we discuss the conditions where we excite the $1^{\text {st }}$ mode. We multiply the secular terms of the $1^{\text {st }}$ mode by $e^{i \zeta_{1}}$ and let $\hat{f}_{1} e^{i r \tau}=$ $\hat{f}_{1} e^{i \sigma T_{1}} e^{i \omega_{1} T_{0}}, \Gamma_{A}=\sigma T_{1}+\zeta_{1}$, and $\Gamma_{B}=3 \zeta_{1}-\zeta_{3}$. The periodic solutions of the beam correspond to the constant solutions (also called fixed points in nonlinear dynamics (see Chapter 5 in Nayfeh and Pai book ${ }^{3}$ ), which correspond to $\Gamma_{A}^{\prime}=\sigma+\zeta_{1}^{\prime}=0, \Gamma_{B}^{\prime}=3 \zeta_{1}^{\prime}-\zeta_{3}^{\prime}=0$, and $\frac{\partial B_{1}}{\partial T_{1}}=\frac{\partial B_{3}}{\partial T_{1}}=0$ in Eq. (23). Thus, the real part can be written as

$$
\begin{aligned}
- & \frac{1}{2} \hat{A}\left[\gamma_{1}^{4}\left(\int_{0}^{1} \cos ^{2} \gamma_{1} x d x\right)\left(3 B_{1} B_{1} \bar{B}_{1}\right)+\right. \\
& \left.\gamma_{1}^{2} \gamma_{3}^{2}\left(\int_{0}^{1} \cos ^{2} \gamma_{3} x d x\right)\left(2 B_{1} B_{3} \bar{B}_{3}\right)\right]+2 \omega_{1} \sigma B_{1}\left(T_{1}\right)- \\
& A_{31} K\left(6 B_{1} B_{3} \bar{B}_{3} \int_{0}^{1} \phi_{1}^{2} \phi_{3}^{2} d x+3 B_{1}^{2} \bar{B}_{1} \int_{0}^{1} \phi_{1}^{4} d x+\right. \\
& \left.3 \bar{B}_{1}^{2} B_{3} \cos \Gamma_{B} \int_{0}^{1} \phi_{1}^{3} \phi_{3} d x\right)=-A_{21} \hat{f}_{1} \cos \Gamma_{A}
\end{aligned}
$$

and the imaginary part as

$$
\begin{array}{r}
-\mu \omega_{1} B_{1}\left(T_{1}\right)-A_{31} K\left(3 \bar{B}_{1}^{2} B_{3} \sin \Gamma_{B} \int_{0}^{1} \phi_{1}^{3} \phi_{3} d x\right)= \\
-A_{21} \hat{f}_{1} \sin \Gamma_{A}
\end{array}
$$

After calculating the sum of the squares of Eqs. (24) and (25) and eliminating the terms associated with time, we obtain the 
following:

$$
\begin{aligned}
\{- & \frac{1}{2} \hat{A}\left[\gamma_{1}^{4}\left(\int_{0}^{1} \cos ^{2} \gamma_{1} x d x\right)\left(3 B_{1} B_{1} \bar{B}_{1}\right)+\right. \\
& \left.\gamma_{1}^{2} \gamma_{3}^{2}\left(\int_{0}^{1} \cos ^{2} \gamma_{3} x d x\right)\left(2 B_{1} B_{3} \bar{B}_{3}\right)\right]+2 \omega_{1} \sigma B_{1}\left(T_{1}\right)- \\
& A_{31} K\left(6 B_{1} B_{3} \bar{B}_{3} \int_{0}^{1} \phi_{1}^{2} \phi_{3}^{2} d x+3 B_{1}^{2} \bar{B}_{1} \int_{0}^{1} \phi_{1}^{4} d x+\right. \\
& \left.\left.3 \bar{B}_{1}^{2} B_{3} \cos \Gamma_{B} \int_{0}^{1} \phi_{1}^{3} \phi_{3} d x\right)\right\}^{2}+\left\{-\mu \omega_{1} B_{1}\left(T_{1}\right)-\right. \\
& \left.A_{31} K\left(3 \bar{B}_{1}^{2} B_{3} \sin \Gamma_{B} \int_{0}^{1} \phi_{1}^{3} \phi_{3} d x\right)\right\}^{2}=A_{21}^{2} \hat{f}_{1}^{2}
\end{aligned}
$$

We then multiple the secular terms of the $3^{\text {rd }}$ mode (Eq. (23)) by $e^{i \zeta_{3}}$. The real part can be written as

$$
\begin{aligned}
& -\frac{1}{2} \hat{A}\left[\gamma_{3}^{4}\left(\int_{0}^{1} \cos ^{2} \gamma_{3} x d x\right)\left(3 B_{3} B_{3} \bar{B}_{3}\right)+\right. \\
& \left.\gamma_{1}^{2} \gamma_{3}^{2}\left(\int_{0}^{1} \cos ^{2} \gamma_{1} x d x\right)\left(2 B_{3} B_{1} \bar{B}_{1}\right)\right]+6 \omega_{3} \sigma B_{3}\left(T_{1}\right)- \\
& A_{33} K\left[\left(B_{1}^{3} \int_{0}^{1} \phi_{1}^{3} \phi_{3} d x\right) \cos \left(-\Gamma_{B}\right)+\right. \\
& \left.6 B_{1} \bar{B}_{1} B_{3} \int_{0}^{1} \phi_{1}^{2} \phi_{3}^{2} d x+3 B_{3}^{2} \bar{B}_{3} \int_{0}^{1} \phi_{3}^{4} d x\right]=0
\end{aligned}
$$

and the imaginary part as

$$
-\mu \omega_{3} B_{3}\left(T_{1}\right)-A_{33} K\left[\left(B_{1}^{3} \int_{0}^{1} \phi_{1}^{3} \phi_{3} d x\right) \sin \left(-\Gamma_{B}\right)\right]=0 .
$$

We solve Eqs. (26) to (28) using numerical methods, which enables us to plot the fixed points plots for amplitudes $B_{1}$ and $B_{3}$ (since the solutions are corresponding to the fixed points of $\frac{\partial B_{1}}{\partial T_{1}}=\frac{\partial B_{3}}{\partial T_{1}}=0$ ) and tuned frequency $\sigma$ in the system in order to observe the internal resonance. Due to limitations regarding the length of the manuscript, we will not go into details of the analysis where we excite the $3^{\text {rd }}$ mode, which is similar to that of the $1^{\text {st }}$ mode.

\subsection{Verification of Internal Resonance}

Using the relationship between amplitude and frequency in the nonlinear system without a DVA, we plotted the fixed point plots to search for 1:3 internal resonance. The left part of Fig. 2 displays the fixed points plots when the $1^{\text {st }}$ mode (lower mode) is excited by a harmonic force. The horizontal axis measures the tuned frequency near the natural frequency in this mode, and the vertical axis measures the amplitude of vibration in the beam. As can be seen in Fig. 2, multiple amplitudes may correspond to a single frequency. A system that resides within this unstable region for long periods of time can undergo fatigue and damage. Figure 3 presents the fixed points plots of the $3^{\text {rd }}$ mode. When the $1^{\text {st }}$ mode is being excited, the amplitudes of the $1^{\text {st }}$ mode (Fig. 2) are greater than those of the $3^{\text {rd }}$ mode (Fig. 3), which is normal. Fixed point plots of the $3^{\text {rd }}$ mode under excitation are presented in the left part of Fig. 4, and the concurrent fixed point plots of the $1^{\text {st }}$ mode are presented in Fig. 5. A comparison of the two revealed that although it was the $3^{\text {rd }}$ mode that was being excited, the amplitudes in the $1^{\text {st }}$ mode were greater than those of the $3^{\text {rd }}$ mode, due to the unique internal resonance associated with nonlinear systems. This proves that 1:3 internal resonance occurs in the $1^{\text {st }}$ and $3^{\text {rd }}$ modes of the system.

\subsection{Numerical Verification}

To ensure the internal resonance in the system, we employed numerical calculations to verify the results in the fixed point plots without the DVA (i.e., the solutions of Eqs. (26) to (28)). Since Eqs. (26) to (28) are the solvability conditions from Eq. (21), we substituted the vibration mode obtained using Eq. (21) into Eq. (13) and employed the orthogonal properties for its integration, the result of which was then used to excite the $1^{\text {st }}$ mode, thus producing

$$
\begin{aligned}
& \stackrel{* *}{\xi}_{1}+\gamma_{1}^{4} \xi_{1}+\mu \stackrel{*}{\xi}_{1}+\bar{\omega}^{2} \xi_{1}+\frac{\hat{k}}{\int_{0}^{1} \phi_{1}^{2} d x} \Gamma_{1}+ \\
& \frac{1}{2} \gamma_{1}^{2} \xi_{1} \int_{0}^{1}\left(\gamma_{1}^{2} \xi_{1}^{2} \cos ^{2} \gamma_{1} x+\gamma_{3}^{2} \xi_{3}^{2} \cos ^{2} \gamma_{3} x\right) d x=\bar{F} \frac{\int_{0}^{1} \phi_{1} d x}{\int_{0}^{1} \phi_{1}^{2} d x}
\end{aligned}
$$

where

$$
\Gamma_{1}=\int_{0}^{1}\left(\xi_{1}^{3} \phi_{1}^{3}+3 \xi_{1}^{2} \phi_{1}^{2} \xi_{3} \phi_{3}+3 \xi_{1} \phi_{1} \xi_{3}^{2} \phi_{3}^{2}+\xi_{3}^{3} \phi_{3}^{3}\right) \phi_{1} d x
$$

When it was used to excite the $3^{\text {rd }}$ mode, the outcome was

$$
\begin{aligned}
& \stackrel{* *}{\xi}_{3}+\gamma_{3}^{4} \xi_{3}+\mu \stackrel{*}{\xi}_{3}+\bar{\omega}^{2} \xi_{3}+\frac{\hat{k}}{\int_{0}^{1} \phi_{3}^{2} d x} \Gamma_{3}+ \\
& \frac{1}{2} \gamma_{3}^{2} \xi_{3} \int_{0}^{1}\left(\gamma_{1}^{2} \xi_{1}^{2} \cos ^{2} \gamma_{1} x+\gamma_{3}^{2} \xi_{3}^{2} \cos ^{2} \gamma_{3} x\right) d x=\bar{F} \frac{\int_{0}^{1} \phi_{3} d x}{\int_{0}^{1} \phi_{3}^{2} d x}
\end{aligned}
$$

where

$$
\Gamma_{3}=\int_{0}^{1}\left(\xi_{1}^{3} \phi_{1}^{3}+3 \xi_{1}^{2} \phi_{1}^{2} \xi_{3} \phi_{3}+3 \xi_{1} \phi_{1} \xi_{3}^{2} \phi_{3}^{2}+\xi_{3}^{3} \phi_{3}^{3}\right) \phi_{3} d x
$$

For the integration coefficients, please refer to Appendix 2. We used the Runge-Kutta (RK4) method to solve the dynamic equations (Eqs. (29) and (31)) and then drew time response graphs and Poincaré maps. The right side of Fig. 2 presents the fixed point plot and the numerical verification graph of the $1^{\text {st }}$ mode; the upper graph displays the time responses, and the lower graph is the Poincaré map. The horizontal axis of a fixed points plot represents the tuned frequency near the beam's natural frequency. Since the external forcing function was defined as $\hat{f}_{m} e^{i r \tau}=\hat{f}_{m} e^{i\left(\omega_{m}+\varepsilon \sigma\right) T_{0}}=\hat{f}_{m}\left(e^{i \varepsilon \sigma T_{0}} e^{i \omega_{m} T_{0}}\right)=$ $\hat{f}_{m} e^{i \sigma T_{1}} e^{i \omega_{m} T_{0}}$, we can see that when $\sigma=0$, the dimensionless forcing frequency $r$ equals the beam's $m^{\text {th }}$ natural frequency $\omega_{m}$. Physically, the fixed point plot displays the nonlinear steady-state (fixed point) frequency response near the system's natural frequencies. The upper graph of Fig. 2 displays the time response graph obtained when $\sigma=4$. The convergence value in this figure is the same as that of the fixed point plot in the left side in Fig. 2 when $\sigma=4$. The Poincaré map also displays unstable in this case (chaos). The right side of 


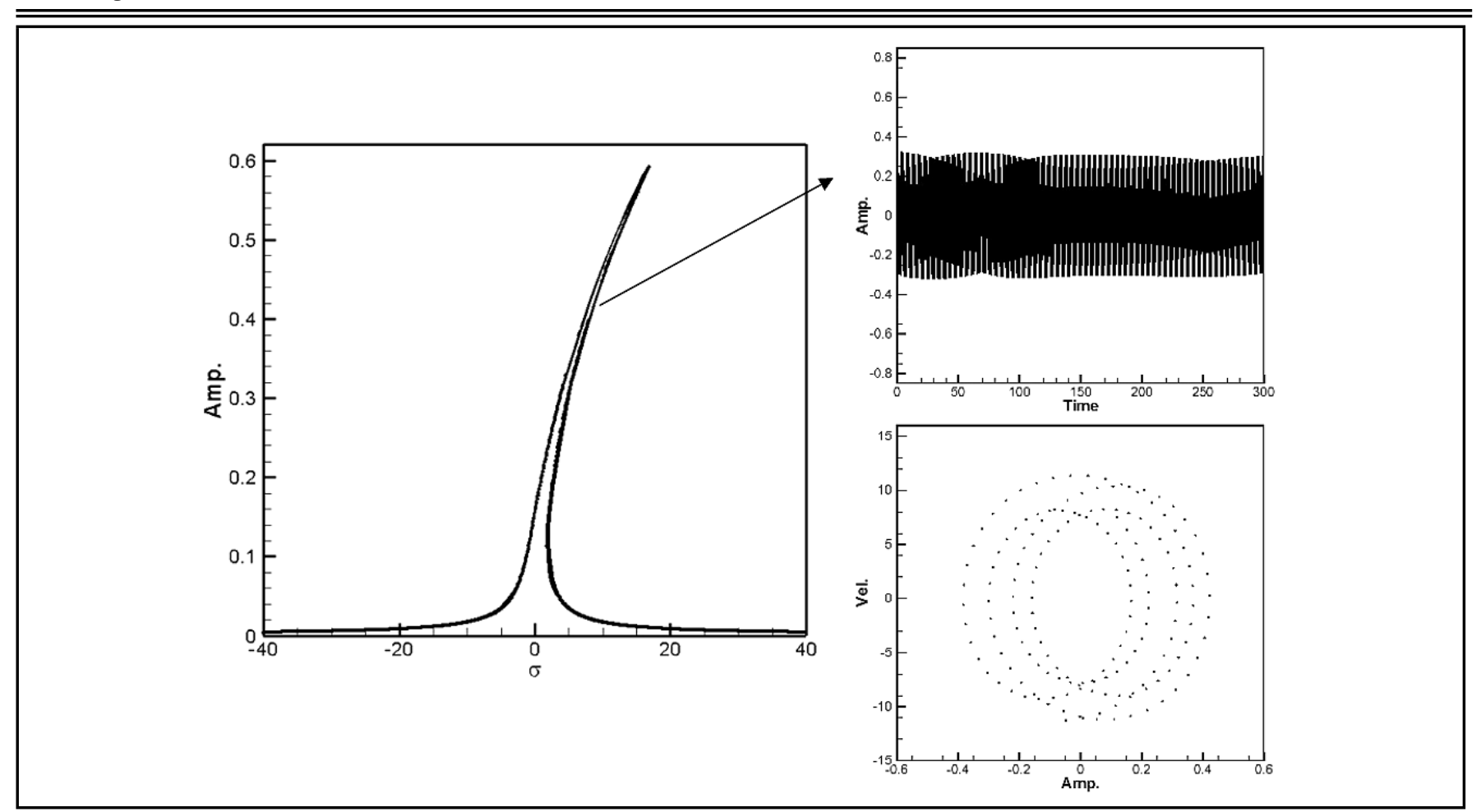

Figure 2. The $1^{\text {st }}$ mode's fixed point plots when the $1^{\text {st }}$ mode is excited (No DVA).

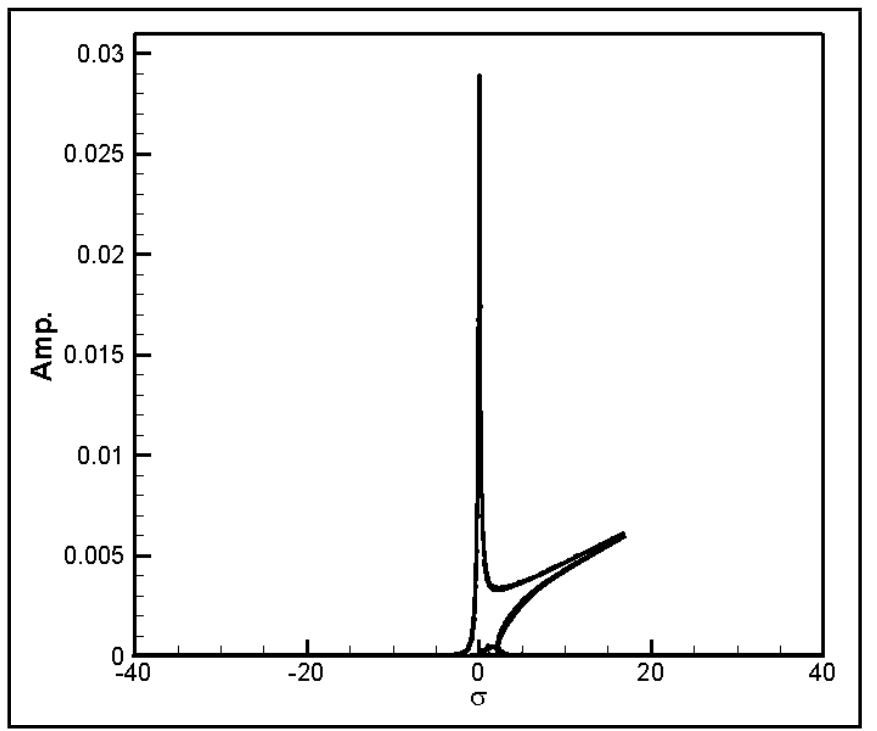

Figure 3. The $3^{\text {rd }}$ mode's fixed point plots when the $1^{\text {st }}$ mode is excited (No DVA).

Fig. 4 presents the fixed point plot and the numerical verification graph of the $3^{\text {rd }}$ mode; again, the upper graph displays the time responses, and the lower graph is the Poincare map for $\sigma=4$. The convergence value of time response is the same as in the left side in Fig. 4 when $\sigma=4$. The Poincaré map also displays unstable in this case (chaos). Both Figs. 2 and 4 show that the internal resonance predicted in the fixed point plots with no DVA is reasonable.

\section{VIBRATION REDUCTION ANALYSIS OF DVA}

This section expands on the results in Sections 2 and 3 by analyzing the damping effects of a DVA on a suspended beam or a beam supported on an elastic foundation. We examined the effects of the DVA fixed at various locations according to the frequency responses of the overall system. The results are presented in Section 5.

\subsection{Analysis of DVA Equation}

We assumed that the DVA motions constitute a single-point force ( $x_{D}$ denotes the location of the DVA). To enhance the influence of the DVA and simplify the setup, we did not consider the damping term of the DVA. Thus, the motion of equation for $\varepsilon^{1}$ can be written as

$$
\begin{aligned}
\varepsilon^{1}: & \frac{\partial^{2} W_{1}}{\partial T_{0}^{2}}+\frac{\partial^{4} W_{1}}{\partial X^{4}}+\bar{\omega}^{2} \bar{W}_{1}= \\
& \frac{\partial^{2} W_{0}}{\partial X^{2}}\left(\frac{1}{2} \int_{0}^{1}\left(\frac{\partial W_{0}}{\partial X}\right)^{2} d x\right)+F-2 \frac{\partial^{2} W_{0}}{\partial T_{0} \partial T_{1}}- \\
& \mu \frac{\partial W_{0}}{\partial T_{0}}-\hat{k} W_{0}^{3}-\widetilde{k}\left(W_{0}(x, t)-W_{D}\right) \delta\left(x-x_{D}\right) .
\end{aligned}
$$

The DVA equation is as follows:

$$
m_{0} \stackrel{* *}{W}_{D}(t)-\widetilde{k}\left[\sum_{m=1}^{\infty} W_{m}(x, t)-W_{D}\right]=0 .
$$

In Section 3, we established that 1:3 internal resonance occurs in the main body between the modes of $m=1$ and $m=3$ when $\bar{\omega}=3 \pi^{2}$. Thus, we only discussed the $1^{\text {st }}$ and the $3^{\text {rd }}$ modes ( $m=1$ and $m=3$ ) of the system. It is noted that the spring force of the DVA is decided by the relative motion of the DVA $\left(W_{D}\right)$ and the beam $\left(W_{m}\right)$. It can be expressed as $\widetilde{k}\left(W_{m}-W_{D}\right)$. By using the Newton's $2^{\text {nd }}$ law, this force can be added on the beam shown in Eq. (33). As the DVA is considered an external force, we first solved the displacement of the DVA and then substituted it into the equation of motion for the main body in Eq. (33). With Eq. (34), let $m=1$ and 


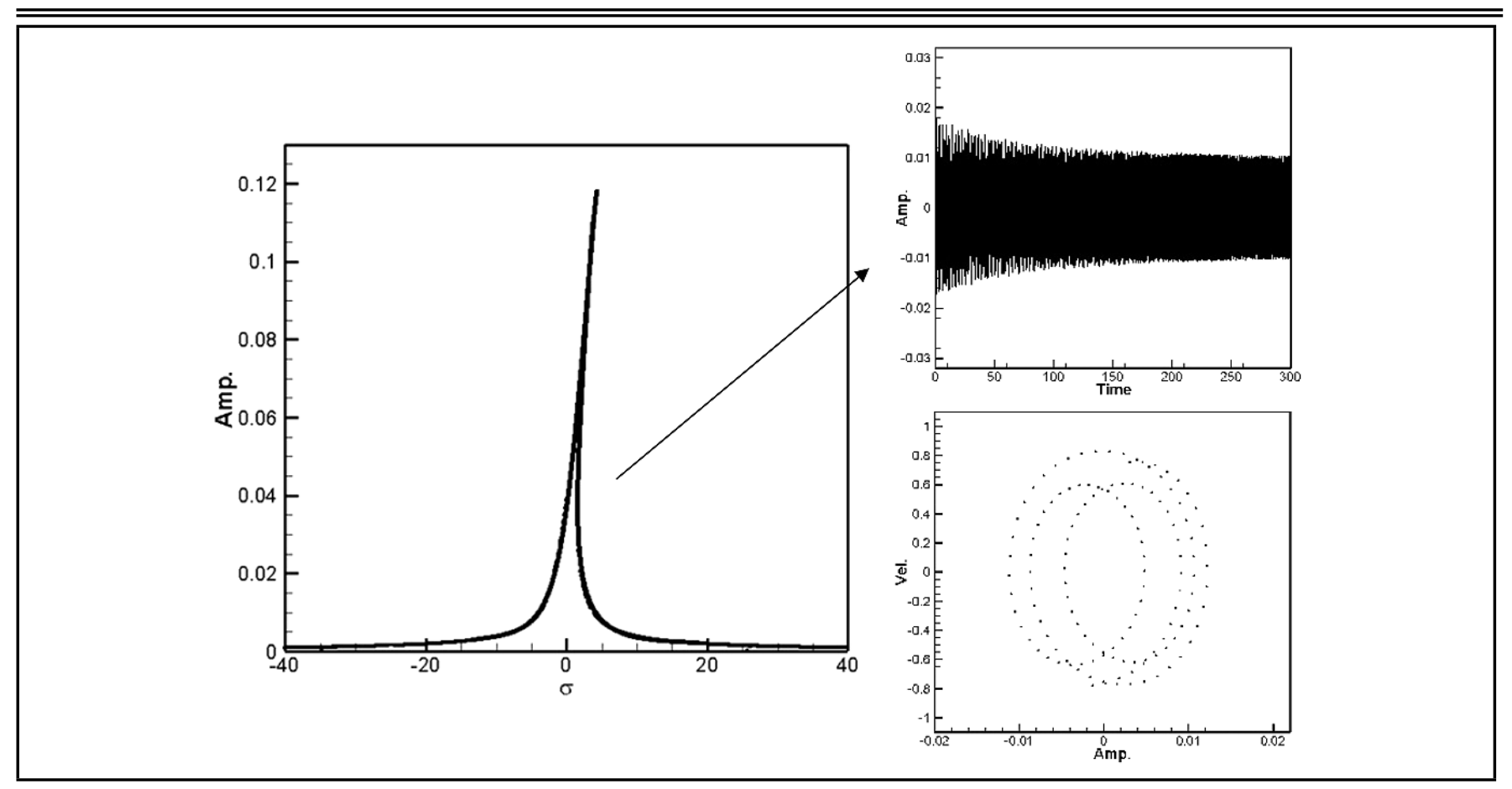

Figure 4. The $3^{\text {rd }}$ mode's fixed point plots when the $3^{\text {rd }}$ mode is excited (No DVA).

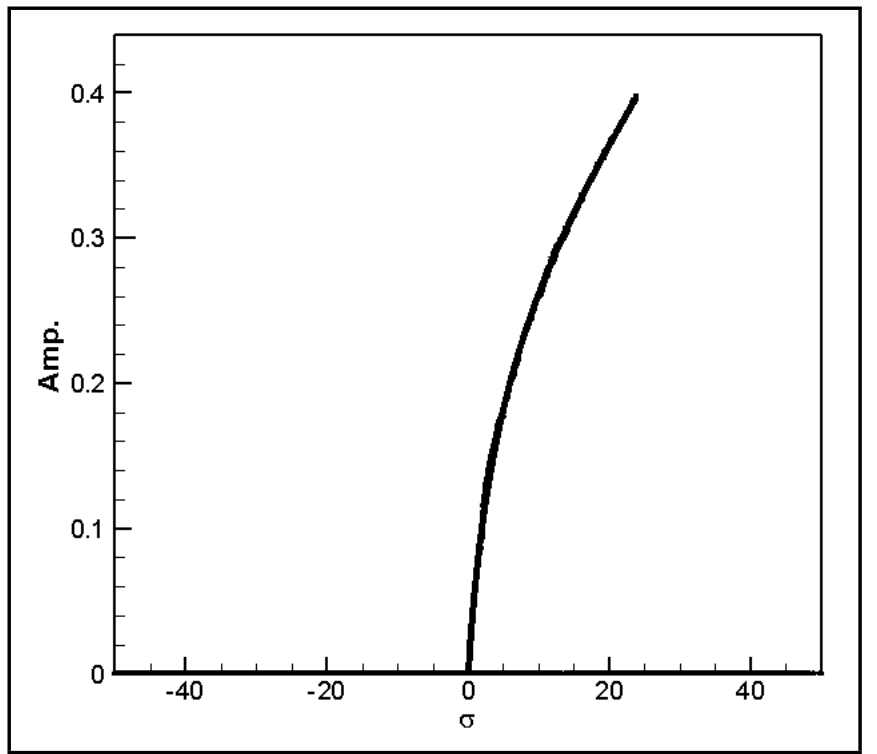

Figure 5. The $1^{\text {st }}$ mode's fixed point plots when the $3^{\text {rd }}$ mode is excited (No DVA).

$m=3$. Then, the equation can be expanded into

$$
m_{0} \stackrel{* *}{W}_{D}(t)-\widetilde{k}\left[\phi_{1}\left(x_{D}\right) \xi_{1}(t)+\phi_{3}\left(x_{D}\right) \xi_{3}(t)-W_{D}\right]=0
$$

Using Eq. (11), we can assume that the solutions for $\xi_{10}$ and $\xi_{30}$ are

$$
\begin{aligned}
& \xi_{10}=B_{1} e^{-i \zeta_{1}} e^{i \omega_{1} T_{0}}+\bar{B}_{1} e^{i \zeta_{1}} e^{-i \omega_{1} T_{0}} \\
& \xi_{30}=B_{3} e^{-i \zeta_{3}} e^{i \omega_{3} T_{0}}+\bar{B}_{3} e^{i \zeta_{3}} e^{-i \omega_{3} T_{0}}
\end{aligned}
$$

which, when substituted into Eq. (35), produce the following:

$$
\begin{gathered}
\stackrel{* *}{W}_{D}+\frac{\widetilde{k}}{m_{0}} W_{D}=\frac{\widetilde{k}}{m_{0}} \phi_{1}\left(x_{D}\right) B_{1} e^{-i \zeta_{1}} e^{i \omega_{1} T_{0}}+ \\
\frac{\widetilde{k}}{m_{0}} \phi_{1}\left(x_{D}\right) \bar{B}_{1} e^{-i \zeta_{1}} e^{-i \omega_{1} T_{0}}+\frac{\widetilde{k}}{m_{0}} \phi_{3}\left(x_{D}\right) B_{3} e^{-i \zeta_{3}} e^{i \omega_{3} T_{0}}+ \\
\frac{\widetilde{k}}{m_{0}} \phi_{3}\left(x_{D}\right) \bar{B}_{3} e^{-i \zeta_{3}} e^{-i \omega_{3} T_{0}}
\end{gathered}
$$

We can assume that the solution to $W_{D}$ is

$$
\begin{aligned}
W_{D}= & C_{1} e^{-i \zeta_{1}} e^{i \omega_{1} T_{0}}+\bar{C}_{1} e^{i \zeta_{1}} e^{-i \omega_{1} T_{0}}+ \\
& C_{3} e^{-i \zeta_{3}} e^{i \omega_{3} T_{0}}+\bar{C}_{3} e^{i \zeta_{3}} e^{-i \omega_{3} T_{0}}
\end{aligned}
$$

After substituting Eq. (38) into Eq. (37) and comparing the coefficients, we can derive the following:

$$
\begin{aligned}
C_{n}= & B_{n} \frac{\widetilde{k}}{m_{0}} \phi_{n}\left(x_{D}\right) /\left(\widetilde{k}-m_{0} \omega_{n}^{2}\right) ; \\
\bar{C}_{n}= & \bar{B}_{n} \frac{\widetilde{k}}{m_{0}} \phi_{n}\left(x_{D}\right) /\left(\widetilde{k}-m_{0} \omega_{n}^{2}\right) ; \quad n=1,3 ; \\
W_{D}= & C_{1} e^{-i \zeta_{1}} e^{i \omega_{1} T_{0}}+\bar{C}_{1} e^{i \zeta_{1}} e^{-i \omega_{1} T_{0}}+ \\
& C_{3} e^{-i \zeta_{3}} e^{i \omega_{3} T_{0}}+\bar{C}_{3} e^{i \zeta_{3}} e^{-i \omega_{3} T_{0}} .
\end{aligned}
$$

Therefore, we can substitute Eqs. (39) and (40) back into Eq. (33) to determine the secular terms.

\subsection{Frequency Response of The Beam System}

As before, we assumed that the external force is evenly distributed: $\hat{f}_{m} e^{i r \tau}=\hat{f}_{m} e^{i\left(\omega_{m}+\varepsilon \sigma\right) T_{0}}=\hat{f}_{m}\left(e^{i \varepsilon \sigma T_{0}} e^{i \omega_{m} T_{0}}\right)=$ $\hat{f}_{m} e^{i \sigma T_{1}} e^{i \omega_{m} T_{0}}$. In terms of the $1^{\text {st }}$ mode $(m=1)$, we selected 
$\omega_{1}$ and $\omega_{3}-2 \omega_{1}$ for the secular terms:

$$
\begin{aligned}
- & \frac{1}{2} \hat{A}\left[\gamma_{1}^{4}\left(\int_{0}^{1} \cos ^{2} \gamma_{1} x d x\right)\left(3 B_{1} B_{1} \bar{B}_{1} e^{-i \zeta_{1}}\right)+\right. \\
& \left.\gamma_{1}^{2} \gamma_{3}^{2}\left(\int_{0}^{1} \cos ^{2} \gamma_{3} x d x\right)\left(2 B_{1} B_{3} \bar{B}_{3} e^{-i \zeta_{1}}\right)\right]+ \\
& A_{21} \bar{F}_{1}-2 i \omega_{1} B_{1}^{\prime}\left(T_{1}\right) e^{-i \zeta_{1}}-2 \omega_{1} \zeta_{1}^{\prime} B_{1}\left(T_{1}\right) e^{-i \zeta_{1}}- \\
& \mu i \omega_{1} B_{1}\left(T_{1}\right) e^{-i \zeta_{1}}-A_{31} K\left(6 B_{1} B_{3} \bar{B}_{3} \int_{0}^{1} \phi_{1}^{2} \phi_{3}^{2} d x e^{-i \zeta_{1}}+\right. \\
& \left.3 B_{1}^{2} \bar{B}_{1} \int_{0}^{1} \phi_{1}^{4} d x e^{-i \zeta_{1}}+3 \bar{B}_{1}^{2} B_{3} \int_{0}^{1} \phi_{1}^{3} \phi_{3} d x e^{i\left(2 \zeta_{1}-\zeta_{3}\right)}\right)- \\
& \widetilde{k}_{1}\left\{B_{1} \phi_{1}\left(x_{D}\right) e^{-i \zeta_{1}}-B_{1} e^{-i \zeta_{1}}\left[\frac{\widetilde{k} \phi_{1}\left(x_{D}\right)}{m_{0}\left(\widetilde{k}-m_{0} \omega_{1}^{2}\right)}\right]\right\}
\end{aligned}
$$

For the $3^{\text {rd }}$ mode $(m=3)$, we selected $\omega_{3}$ and $3 \omega_{1}$ as the secular terms:

$$
\begin{aligned}
& -\frac{1}{2} \hat{A}\left[\gamma_{3}^{4}\left(\int_{0}^{1} \cos ^{2} \gamma_{3} x d x\right)\left(3 B_{3} B_{3} \bar{B}_{3} e^{-i \zeta_{3}}\right)+\right. \\
& \left.\gamma_{1}^{2} \gamma_{3}^{2}\left(\int_{0}^{1} \cos ^{2} \gamma_{1} x d x\right)\left(2 B_{3} B_{1} \bar{B}_{1} e^{-i \zeta_{3}}\right)\right]+ \\
& A_{23} \bar{F}_{3}-2 i \omega_{3} B_{3}^{\prime}\left(T_{1}\right) e^{-i \zeta_{3}}-2 \omega_{3} \zeta_{3}^{\prime} B_{3}\left(T_{1}\right) e^{-i \zeta_{3}}- \\
& \mu i \omega_{3} B_{3}\left(T_{1}\right) e^{-i \zeta_{3}}-A_{33} K\left(B_{1}^{3} \int_{0}^{1} \phi_{1}^{3} \phi_{3} d x e^{-i 3 \zeta_{1}}+\right. \\
& \left.6 B_{1} \bar{B}_{1} B_{3} \int_{0}^{1} \phi_{1}^{2} \phi_{3}^{2} d x e^{-i \zeta_{3}}+3 B_{3}^{2} \bar{B}_{3} \int_{0}^{1} \phi_{3}^{4} d x e^{-i \zeta_{3}}\right)- \\
& \widetilde{k}_{3}\left\{B_{3} \phi_{3}\left(x_{D}\right) e^{-i \zeta_{3}}-B_{3} e^{-i \zeta_{3}}\left[\frac{\widetilde{k} \phi_{3}\left(x_{D}\right)}{m_{0}\left(\widetilde{k}-m_{0} \omega_{3}^{2}\right)}\right]\right\}
\end{aligned}
$$

We then designated that the secular terms are equal to 0 in order to derive a solvability condition. Below, we discuss the circumstances when an external force excites the $1^{\text {st }}$ mode $\left(\hat{f}_{1} e^{i r \tau}=\hat{f}_{1} e^{i \sigma T_{1}} e^{i \omega_{1} T_{0}}\right)$. We multiplied all of the secular terms of the $1^{\text {st }}$ mode by $e^{i \zeta_{1}}$ and let $\Gamma_{A}=\sigma T_{1}+\zeta_{1}$ and $\Gamma_{B}=3 \zeta_{1}-\zeta_{3}$. The real part can be written as

$$
\begin{aligned}
& -\frac{1}{2} \hat{A}\left[\gamma_{1}^{4}\left(\int_{0}^{1} \cos ^{2} \gamma_{1} x d x\right)\left(3 B_{1} B_{1} \bar{B}_{1}\right)+\right. \\
& \left.\gamma_{1}^{2} \gamma_{3}^{2}\left(\int_{0}^{1} \cos ^{2} \gamma_{3} x d x\right)\left(2 B_{1} B_{3} \bar{B}_{3}\right)\right]-2 \omega_{1} \zeta_{1}^{\prime} B_{1}\left(T_{1}\right)- \\
& A_{31} K\left(6 B_{1} B_{3} \bar{B}_{3} \int_{0}^{1} \phi_{1}^{2} \phi_{3}^{2} d x+3 B_{1}^{2} \bar{B}_{1} \int_{0}^{1} \phi_{1}^{4} d x+\right. \\
& \left.3 \bar{B}_{1}^{2} B_{3} \cos \Gamma_{B} \int_{0}^{1} \phi_{1}^{3} \phi_{3} d x\right)-\widetilde{k}_{1} B_{1} \phi_{1}\left(x_{D}\right)+ \\
& B_{1} \frac{\widetilde{k}_{1} \widetilde{k} \phi_{1}\left(x_{D}\right)}{m_{0}\left(\widetilde{k}-m_{0} \omega_{1}^{2}\right)}=-A_{21} \hat{f}_{1} \cos \Gamma_{A}
\end{aligned}
$$

and the imaginary part can be written as

$$
\begin{aligned}
& -2 \omega_{1} B_{1}^{\prime}\left(T_{1}\right)-\mu \omega_{1} B_{1}\left(T_{1}\right)- \\
& A_{31} K\left(3 \bar{B}_{1}^{2} B_{3} \sin \Gamma_{B} \int_{0}^{1} \phi_{1}^{3} \phi_{3} d x\right)=-A_{21} \hat{f}_{1} \sin \Gamma_{A} .
\end{aligned}
$$

We multiplied all of the secular terms of the $3^{\text {rd }}$ mode by $e^{i \zeta_{3}}$. The real part can be written as

$$
\begin{aligned}
& -\frac{1}{2} \hat{A}\left[\gamma_{3}^{4}\left(\int_{0}^{1} \cos ^{2} \gamma_{3} x d x\right)\left(3 B_{3} B_{3} \bar{B}_{3}\right)+\right. \\
& \left.\gamma_{1}^{2} \gamma_{3}^{2}\left(\int_{0}^{1} \cos ^{2} \gamma_{1} x d x\right)\left(2 B_{3} B_{1} \bar{B}_{1}\right)\right]-2 \omega_{3} \zeta_{3}^{\prime} B_{3}\left(T_{1}\right)- \\
& A_{33} K\left[\left(B_{1}^{3} \int_{0}^{1} \phi_{1}^{3} \phi_{3} d x\right) \cos \left(-\Gamma_{B}\right)+\right. \\
& \left.6 B_{1} \bar{B}_{1} B_{3} \int_{0}^{1} \phi_{1}^{2} \phi_{3}^{2} d x+3 B_{3}^{2} \bar{B}_{3} \int_{0}^{1} \phi_{3}^{4} d x\right]- \\
& \widetilde{k}_{3} B_{3} \phi_{3}\left(x_{D}\right)+B_{3} \frac{\widetilde{k}_{3} \widetilde{k} \phi_{3}\left(x_{D}\right)}{m_{0}\left(\widetilde{k}-m_{0} \omega_{3}^{2}\right)}=0
\end{aligned}
$$

and the imaginary part written as

$$
\begin{aligned}
& -2 \omega_{3} B_{3}^{\prime}\left(T_{1}\right)-\mu \omega_{3} B_{3}\left(T_{1}\right)- \\
& A_{33} K\left[\left(B_{1}^{3} \int_{0}^{1} \phi_{1}^{3} \phi_{3} d x\right) \sin \left(-\Gamma_{B}\right)\right]=0 .
\end{aligned}
$$

To obtain the frequency responses of the system at the fixed points, we let $\Gamma_{A}^{\prime}=\sigma+\zeta_{1}^{\prime}=0 \Longrightarrow \zeta_{1}^{\prime}=-\sigma$, $\Gamma_{B}^{\prime}=3 \zeta_{1}^{\prime}-\zeta_{3}^{\prime}=0 \Longrightarrow \zeta_{3}^{\prime}=-3 \sigma$, and $\frac{\partial B_{1}}{\partial T_{1}}=\frac{\partial B_{3}}{\partial T_{1}}=0$ before substituting them into the solvability condition. After calculating the sum of the squares of the real and imaginary portions of the solvability conditions for the $1^{\text {st }}$ mode and eliminating the terms associated with time, we obtain

$$
\begin{aligned}
\{- & \frac{1}{2} \hat{A}\left[\gamma_{1}^{4}\left(\int_{0}^{1} \cos ^{2} \gamma_{1} x d x\right)\left(3 B_{1} B_{1} \bar{B}_{1}\right)+\right. \\
& \left.\gamma_{1}^{2} \gamma_{3}^{2}\left(\int_{0}^{1} \cos ^{2} \gamma_{3} x d x\right)\left(2 B_{1} B_{3} \bar{B}_{3}\right)\right]+2 \omega_{1} \sigma B_{1}\left(T_{1}\right)- \\
& A_{31} K\left(6 B_{1} B_{3} \bar{B}_{3} \int_{0}^{1} \phi_{1}^{2} \phi_{3}^{2} d x+3 B_{1}^{2} \bar{B}_{1} \int_{0}^{1} \phi_{1}^{4} d x+\right. \\
& \left.3 \bar{B}_{1}^{2} B_{3} \cos \Gamma_{B} \int_{0}^{1} \phi_{1}^{3} \phi_{3} d x\right)-\widetilde{k}_{1} B_{1} \phi_{1}\left(x_{D}\right)+ \\
& \left.B_{1} \frac{\widetilde{k}_{1} \widetilde{k} \phi_{1}\left(x_{D}\right)}{m_{0}\left(\widetilde{k}-m_{0} \omega_{1}^{2}\right)}\right\}^{2}+\left\{-\mu \omega_{1} B_{1}\left(T_{1}\right)-\right. \\
& \left.A_{31} K\left(3 \bar{B}_{1}^{2} B_{3} \sin \Gamma_{B} \int_{0}^{1} \phi_{1}^{3} \phi_{3} d x\right)\right\}^{2}=A_{21}^{2} \hat{f}_{1}^{2}
\end{aligned}
$$

The real part of the solvability conditions for the $3^{\text {rd }}$ mode is

$$
\begin{aligned}
& -\frac{1}{2} \hat{A}\left[\gamma_{3}^{4}\left(\int_{0}^{1} \cos ^{2} \gamma_{3} x d x\right)\left(3 B_{3} B_{3} \bar{B}_{3}\right)+\right. \\
& \left.\gamma_{1}^{2} \gamma_{3}^{2}\left(\int_{0}^{1} \cos ^{2} \gamma_{1} x d x\right)\left(2 B_{3} B_{1} \bar{B}_{1}\right)\right]+6 \omega_{3} \sigma B_{3}\left(T_{1}\right)- \\
& \widetilde{k}_{3} B_{3} \phi_{3}\left(x_{D}\right)-A_{33} K\left[\left(B_{1}^{3} \int_{0}^{1} \phi_{1}^{3} \phi_{3} d x\right) \cos \left(-\Gamma_{B}\right)+\right. \\
& \left.6 B_{1} \bar{B}_{1} B_{3} \int_{0}^{1} \phi_{1}^{2} \phi_{3}^{2} d x+3 B_{3}^{2} \bar{B}_{3} \int_{0}^{1} \phi_{3}^{4} d x\right]+ \\
& B_{3} \frac{\widetilde{k}_{3} \tilde{k} \phi_{3}\left(x_{D}\right)}{m_{0}\left(\widetilde{k}-m_{0} \omega_{3}^{2}\right)}=0
\end{aligned}
$$




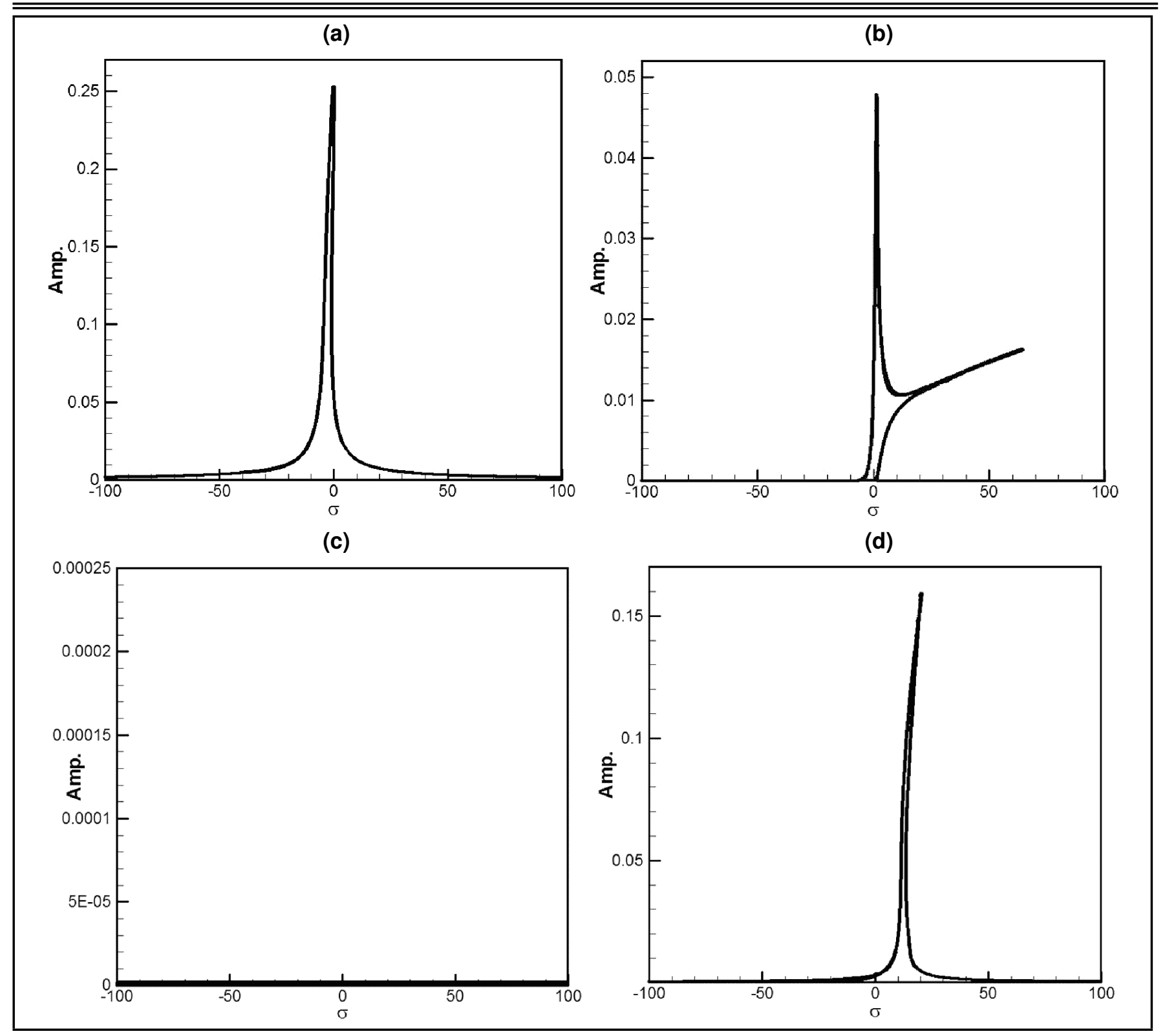

Figure 6. Fixed point plots (with DVA), (a) The $1^{\text {st }}$ mode's plot, when the $1^{\text {st }}$ mode is excited, (b) The $3^{\text {rd }}$ mode's plot, when the $1^{\text {st }}$ mode is excited, (c) The $1^{\text {st }}$ mode's plot, when the $3^{\text {rd }}$ mode is excited, and (d) The $3^{\text {rd }}$ mode's plot, when the $3^{\text {rd }}$ mode is excited.

and the imaginary part is

$$
-\mu \omega_{3} B_{3}\left(T_{1}\right)-A_{33} K\left[\left(B_{1}^{3} \int_{0}^{1} \phi_{1}^{3} \phi_{3} d x\right) \sin \left(-\Gamma_{B}\right)\right]=0 .
$$

Finally, we combine Eqs. (47) to (49) to get the numerical solutions. We can thus draw fixed-point plots for amplitudes $B_{1}$ and $B_{3}$ and $\sigma$ in order to observe the frequency responses. Due to limitations regarding the length of the manuscript, we will not go into details of the analysis of the $3^{\text {rd }}$ mode, which is similar to that of the $1^{\text {st }}$ mode.

\section{RESULTS AND DISCUSSION}

\subsection{Internal Resonance Analysis of Beam System}

Internal resonance occurs in vibration systems with modal frequencies that are multiple integers of each other. When two modes are strongly coupled in cases where internal resonance exists, energy is transferred continuously during motion. The case of no DVA we considered in Figs. 4 and 5, when the $3^{\text {rd }}$ mode is excited, the $1^{\text {st }}$ mode's amplitude is larger than the $3^{\text {rd }}$ mode. This is a typical internal resonance phenomenon in a nonlinear system. In the works of Wang and $\mathrm{Kuo}^{17}$ and Wang and $\mathrm{Tu}^{18}$ for the hinged-free and fixed-free beams, the $1: 3$ internal resonance was found in the $1^{\text {st }}$ and the $2^{\text {nd }}$ modes. In the present study, a different boundary condition was examined for a hinged-hinged nonlinear beam and a 1:3 internal resonance was found in the $1^{\text {st }}$ and the $3^{\text {rd }}$ modes. The elastic foundation dimensionless spring constant was found analytically to be $9 \pi^{4}$ to trig the internal resonance. The effects of various beam boundary conditions in the internal resonance were shown evidently and should be studied individually. Attaching a TMD introduces an additional frequency between the two internal resonant modes to the beam-dampened system, which breaks the multiple integer frequency relationship and thereby mitigates the exchange of energy between the two cou- 


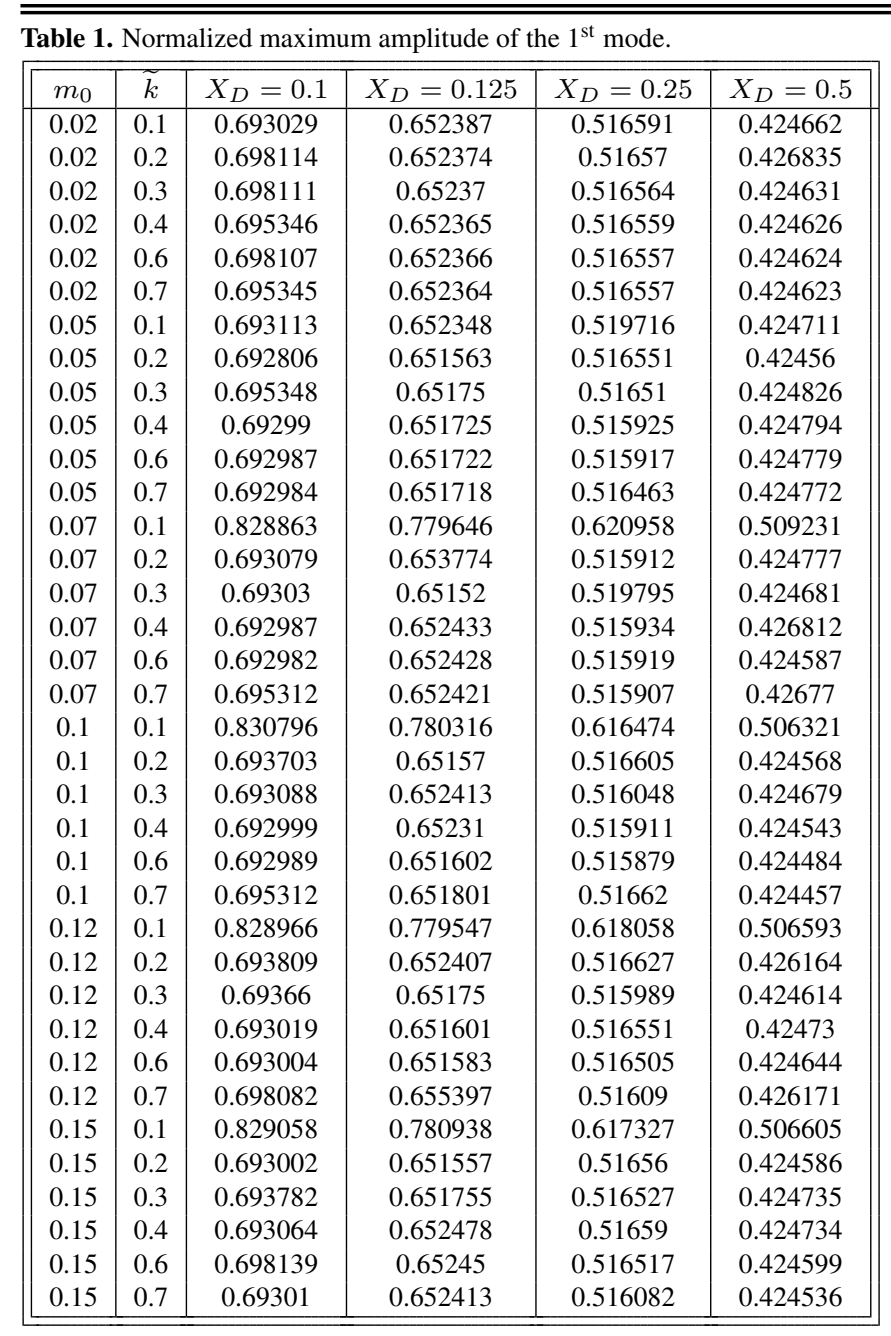

pled modes, thus resulting in a reduction in internal resonance. We employed numerical analysis to solve Eqs. (47) through (49), thereby obtaining the fixed points plots of the system. Figure 6 (the fixed point plots including the DVA) shows that when the $3^{\text {rd }}$ mode is excited, the amplitudes of the $1^{\text {st }}$ mode (Fig. 6c) do not exceed those of the $3^{\text {rd }}$ mode (Fig. 6d). Clearly, internal resonance does not occur. By comparing Fig. 6 with Figs. 2 through 5, we can see that the inclusion of the DVA in the system prevents 1:3 internal resonance in the $1^{\text {st }}$ and $3^{\text {rd }}$ modes. It is possible that the damping effects of the DVA are directly associated with the vibrations of the main beam. In Eq. (7), the term $\widetilde{k}\left[W(x, \tau)-W_{D}\right] \delta\left[x-l_{D}\right]$ couples with $W(x, t)$, which then merges with terms $W^{i v}$ and $\bar{w}^{2}$ to influence (positively or negatively) the previous integer ratio of the frequencies, 1:3. As a result, 1:3 internal resonance does not occur.

\subsection{Analyzing The Effectiveness of DVA Damping}

Using MOMS, eigen analysis, and fixed point plots, we established that 1:3 internal resonance occurs in the $1^{\text {st }}$ and $3^{\text {rd }}$ modes of a main body without a DVA. A comprehensive view of the two modes show that regardless of whether the $1^{\text {st }}$ or $3^{\text {rd }}$ mode is excited, the maximum amplitudes in the $1^{\text {st }}$ and $3^{\text {rd }}$ modes are 0.598675 and 0.11921 , respectively. We thus used these amplitudes as the basis for comparisons used to evaluate the effectiveness of damping. In previous sections, we already
Table 2. Normalized maximum amplitude of the $3^{\text {rd }}$ mode.

\begin{tabular}{|c|c|c|c|c|c|}
\hline$m_{0}$ & $\widetilde{k}$ & $X_{D}=0.1$ & $X_{D}=0.125$ & $X_{D}=0.25$ & $X_{D}=0.5$ \\
\hline 0.02 & 0.1 & 1.464852 & 1.728147 & 1.478419 & 1.778185 \\
0.02 & 0.2 & 1.07284 & 1.229764 & 1.638418 & 2.274189 \\
0.02 & 0.3 & 1.467375 & 1.726061 & 1.78791 & 2.119784 \\
0.02 & 0.4 & 1.467676 & 1.725644 & 2.104751 & 2.481469 \\
0.02 & 0.6 & 1.027626 & 1.228464 & 2.177923 & 2.555767 \\
0.02 & 0.7 & 1.417364 & 1.725503 & 1.597176 & 2.685515 \\
0.05 & 0.1 & 1.40992 & 1.770635 & 1.378139 & 1.668663 \\
0.05 & 0.2 & 1.026374 & 1.26479 & 1.392248 & 1.772926 \\
0.05 & 0.3 & 1.439894 & 1.747833 & 1.431815 & 1.72715 \\
0.05 & 0.4 & 1.442745 & 1.74515 & 1.531946 & 1.839284 \\
0.05 & 0.6 & 1.018009 & 1.255225 & 1.556726 & 1.864086 \\
0.05 & 0.7 & 1.444224 & 1.744312 & 1.597176 & 2.048476 \\
0.07 & 0.1 & 1.055878 & 1.606127 & 2.179459 & 2.278483 \\
0.07 & 0.2 & 1.404675 & 1.296659 & 1.34855 & 1.663102 \\
0.07 & 0.3 & 1.416868 & 1.765247 & 1.357211 & 1.646024 \\
0.07 & 0.4 & 1.423382 & 1.759675 & 1.39794 & 1.691371 \\
0.07 & 0.6 & 0.966909 & 1.274386 & 1.409035 & 1.702337 \\
0.07 & 0.7 & 1.426958 & 1.757671 & 1.427694 & 1.722562 \\
0.1 & 0.1 & 0.004963 & 0.001511 & 0.00313 & 0.001322 \\
0.1 & 0.2 & 0.836822 & 1.365858 & 1.33042 & 1.579634 \\
0.1 & 0.3 & 1.373034 & 1.797928 & 1.304739 & 1.588934 \\
0.1 & 0.4 & 1.389869 & 1.783871 & 1.290711 & 1.573621 \\
0.1 & 0.6 & 0.927665 & 1.305452 & 1.28927 & 1.572192 \\
0.1 & 0.7 & 1.398384 & 1.779369 & 1.287593 & 1.570295 \\
0.12 & 0.1 & 1.458676 & 1.184319 & 1.436823 & 1.59492 \\
0.12 & 0.2 & 0.799082 & 1.437811 & 1.339805 & 1.548611 \\
0.12 & 0.3 & 1.334119 & 1.824971 & 1.288543 & 1.571323 \\
0.12 & 0.4 & 1.363762 & 1.801805 & 1.295016 & 1.527046 \\
0.12 & 0.6 & 0.891536 & 1.327901 & 1.240718 & 1.520059 \\
0.12 & 0.7 & 1.377175 & 1.794745 & 1.229752 & 1.508142 \\
0.15 & 0.1 & 1.33262 & 1.341661 & 1.690585 & 1.822654 \\
0.15 & 0.2 & 1.003977 & 1.6405 & 1.401304 & 1.521638 \\
0.15 & 0.3 & 1.25403 & 1.879512 & 1.28088 & 1.563133 \\
0.15 & 0.4 & 1.316419 & 1.832141 & 1.20584 & 1.481179 \\
0.15 & 0.6 & 0.887034 & 1.364301 & 1.192195 & 1.467781 \\
0.15 & 0.7 & 1.341841 & 1.819752 & 1.17053 & 1.444635 \\
\hline & & & & & \\
\hline
\end{tabular}

established that adding a DVA with another DOF to the model damages the natural vibration frequency ratios of the various modes, due to the fact that the additional DOF couples with the equation of motion of the beam. As a result, internal resonance does not occur. In this section, we examine various parameters of the DVA, including mass ratio, spring constant ratio, and location, to identify the combination with the optimal damping effects. Using the solvability condition and fixed point plots in Section 4 , we derived the maximum amplitude in the $1^{\text {st }}$ mode when excited, and then normalized the results using the maximum amplitude obtained in cases without a DVA (0.598675). To achieve this, we considered the mass ratio (the mass of the DVA / the mass of the beam) and the spring constant ratio (the spring constant of the DVA / the spring constant of the elastic foundation). The normalized maximum amplitudes of the $1^{\text {st }}$ mode are shown in Table 1 . Similarly, we normalized the maximum amplitude in the $3^{\text {rd }}$ mode when excited by using the maximum amplitude in cases without a DVA (0.11921). Again, the results are shown in Table 2. To avoid the difficulties associated with an excessive number of DVA parameters in the tables, we used 3D maximum amplitude projections (3DMACPs) to reveal the influence of parameters on damping performance. The results of 3DMACPs will be shown in next two paragraphs.

This study uses the $3 \mathrm{D}$ projections to project the maximum amplitudes of various parameter combinations onto the massspring constant plane and combine various locations into a 3D maximum amplitude contour plot (3DMACP). Physically, the 


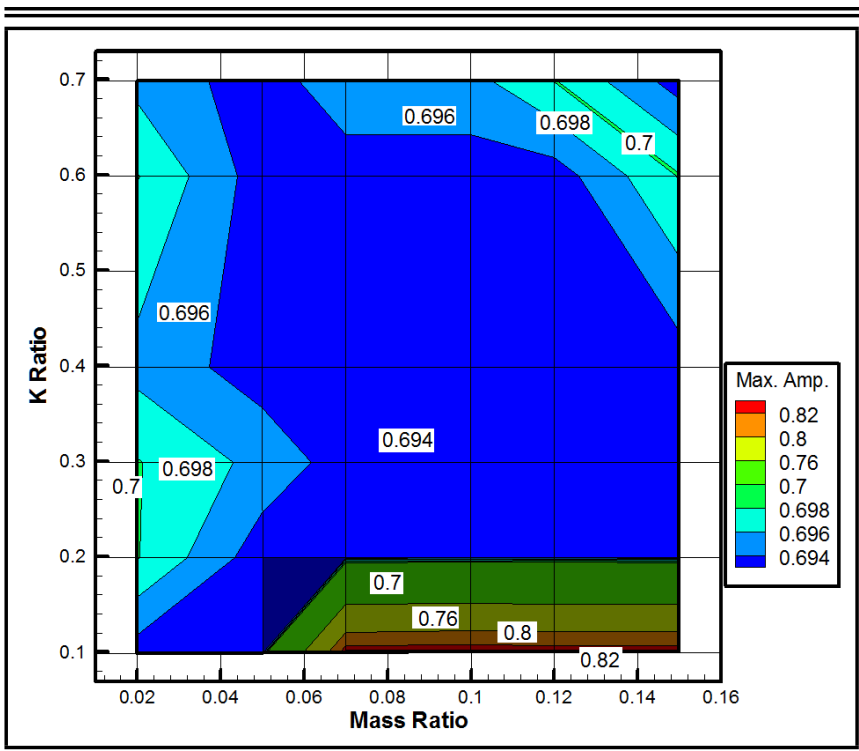

Figure 7. A $3 \mathrm{D}$ MACP chart of the $1^{\text {st }}$ mode, $X_{D}=0.1$.

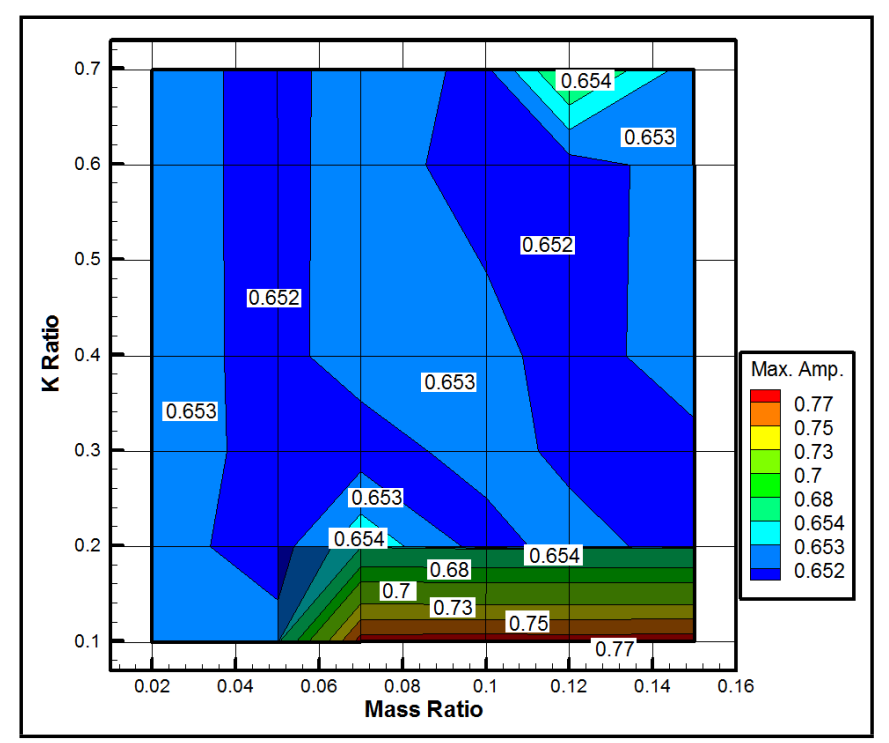

Figure 8. A $3 \mathrm{D}$ MACP chart of the $1^{\text {st }}$ mode, $X_{D}=0.125$.

3DMACPs provide the information regarding the maximum amplitudes of each combination of DVA's mass ratio $\left(m_{0}\right)$ and dimensionless spring constant $(\widetilde{k})$ for a fixed DVA location $\left(X_{D}\right)$. Different colors of the contour levels represent different amplitude values. This is a novel technique to display a set of complicated data and display a rather simple plot for better understanding. Figures 7 through 10 present the 3DMACPs containing the normalized maximum amplitudes of the $1^{\text {st }}$ mode when the $1^{\text {st }}$ mode is excited and the DVA is at $X_{D}=0.1$, $0.125,0.25$, and 0.5 , respectively. To differentiate the damping effects of the DVA at various locations, we drew the contour of the four above combinations with the amplitude ranging from 0.425 to 0.82 . Comparison of Figs. 7 through 10 shows that the effectiveness of the DVA increased as it approached the center of the beam. Furthermore, we selected the mass ratios between 0.02 and 0.15 because if the mass ratio were too small, it would have no effect on the hinged beams. In most civil engineering or mechanical structures, a mass ratio greater than 0.15 would mean an excessive waste of materials on the DVA. In addition, the spring constants considered in this study ranged from 0.1

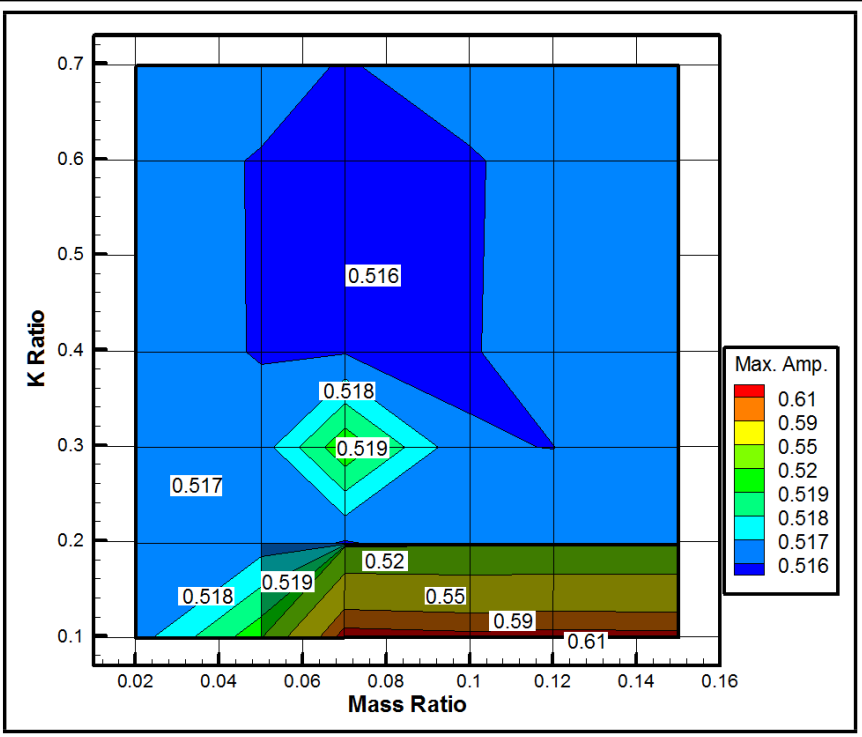

Figure 9. A 3D MACP chart of the $1^{\text {st }}$ mode, $X_{D}=0.25$.

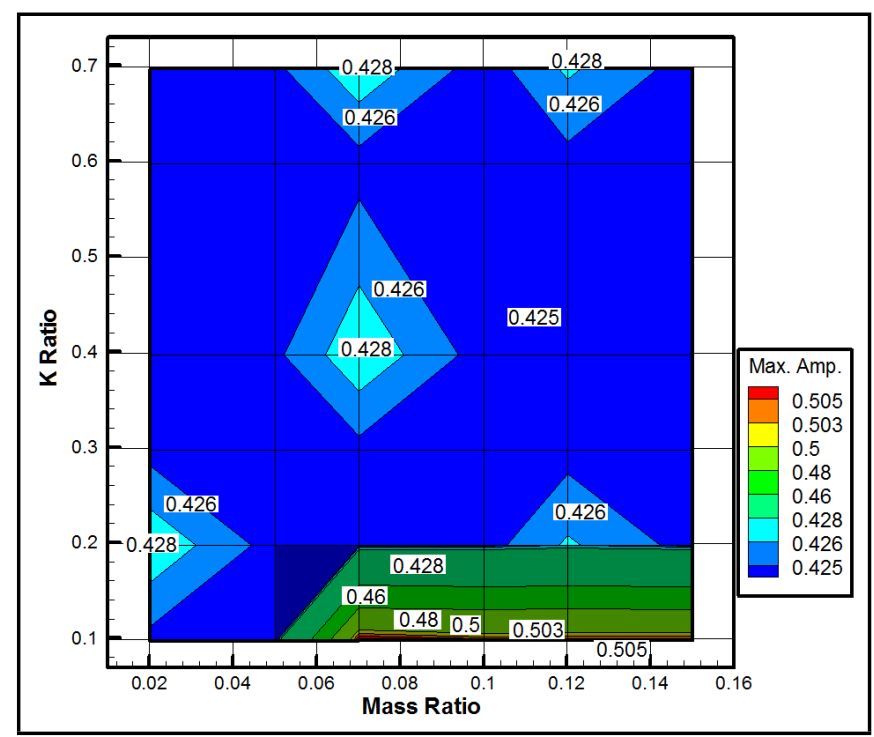

Figure 10. A 3D MACP chart of the $1^{\text {st }}$ mode, $X_{D}=0.5$.

and 0.7 , in accordance with the function proposed by Samani and Pellicano ${ }^{15}$ for the optimal DVA parameter combination of linear hinged-hinged beams:

$$
\widetilde{k}=m_{0}\left(\frac{\omega_{1}}{1+\mu}\right)^{2} ; \quad m_{e}=\frac{m L}{2 \sin ^{2}(\pi d / L)} ; \quad \mu=m_{0} / m_{e}
$$

According to Samani and Pellicano, ${ }^{15}$ we can convert the DVA mass ratio range from 0.02 to 0.15 into the elastic constant ratio estimated by Samani and Pellicano. ${ }^{15}$ The range of $m_{0}$ in this study roughly corresponds to $\widetilde{k}$ values between 0.4 and 0.55. However, the function in Eq. (50) is applicable to only linear beams, as it does not consider the effects of stretching. This effect can cause additional amplitudes in the transverse direction of the beam. In theory, this situation would require a DVA with greater damping effects in order to achieve acceptable results. We therefore widened the range of the elastic constant ratio from $0.4 \sim 0.55$ to $0.1 \sim 0.7$. As for the amplitudes in the $1^{\text {st }}$ mode when the $1^{\text {st }}$ mode is excited, Figs. 7 through 10 show that the DVA being more effective when it is closer 


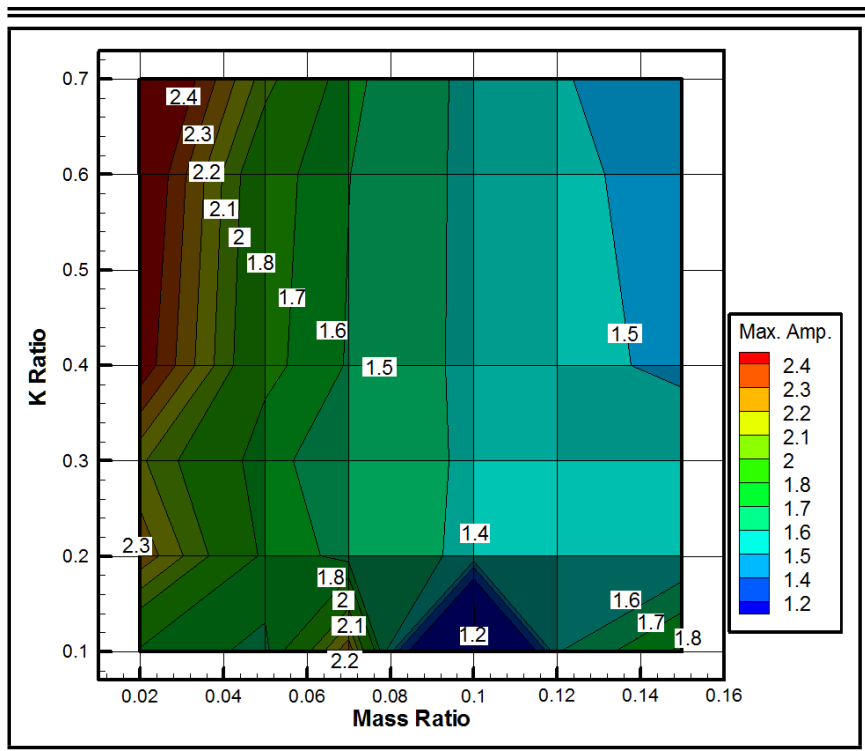

Figure 11. A 3D MACP chart of the $3^{\text {rd }}$ mode, $X_{D}=0.1$.

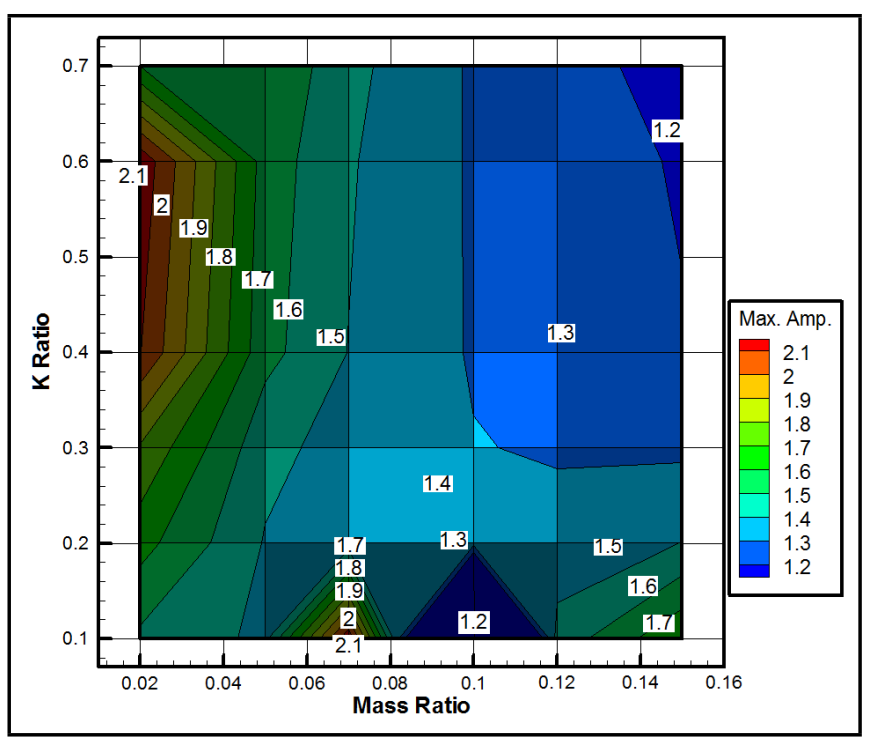

Figure 12. A $3 D$ MACP chart of the $3^{\text {rd }}$ mode, $X_{D}=0.125$.

to the center of the beam a higher mass ratios do not actually have significant damping effects. On the contrary, excessively high mass ratios can magnify the amplitudes in transverse vibrations in the beam (because a DVA is included). We also found that such DVA designs are more effective in damping the $1^{\text {st }}$ mode. Similarly, damping effects are better when the DVA is closer to the center of the beam. Nonetheless, combinations with $m_{0}$ between 0.05 and 0.15 and $\widetilde{k}$ less than 0.2 must still be avoided.

For the $3^{\text {rd }}$ mode (Figures 11 through 14), we created 3DMACPs for the various locations $X_{D}$ and set the range of the maximum amplitude to between 1.0 and 2.4. Note that a color closer to light green indicates that the maximum amplitude is greater than 1 and that the damping effect is worse than if there were no DVA. We also discovered that with mass ratios of approximately 0.1 and elastic constants less than 0.2 , minimum amplitudes occur at each location. This means that said combinations have optimal damping effects in terms of the $3^{\text {rd }}$ mode. In Figs. 7 through 10, we can also see that near the end $\left(X_{D}=0.1\right.$ in Fig. 7$)$, a smaller $m_{0}$ and a greater $\widetilde{k}$

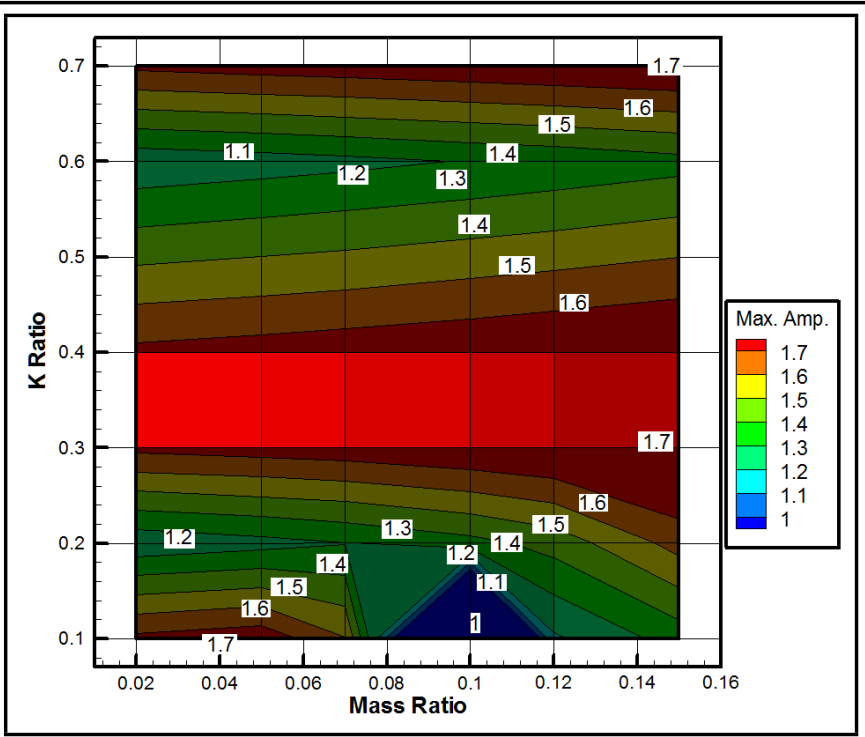

Figure 13. A $3 D$ MACP chart of the $3^{\text {rd }}$ mode, $X_{D}=0.25$.

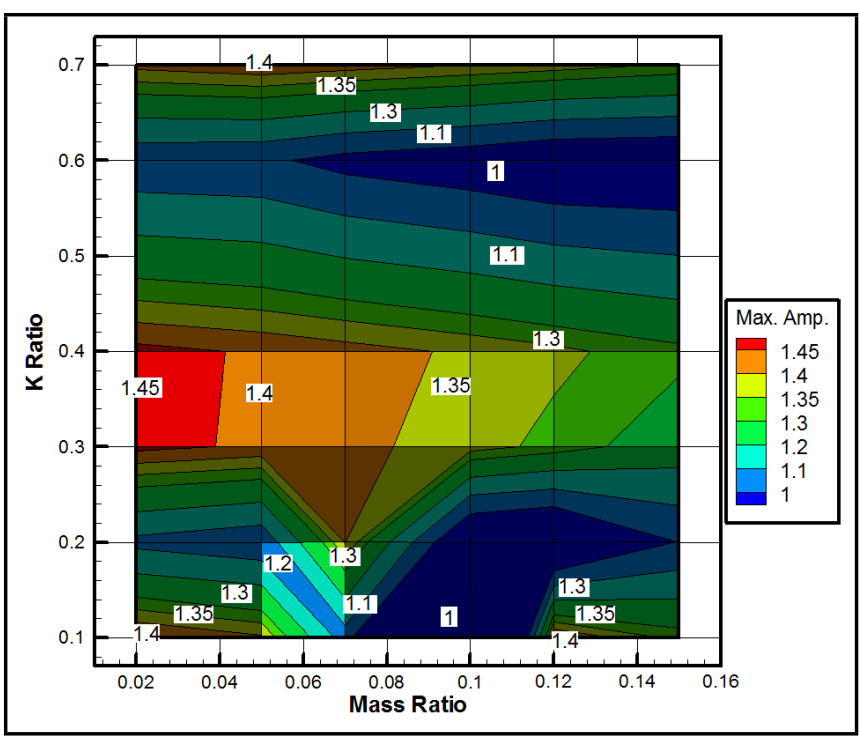

Figure 14. A $3 D$ MACP chart of the $3^{\text {rd }}$ mode, $X_{D}=0.5$.

contribute to poorer damping performance. Of course, placing the DVA near the center of the beam is most effective, but for the $3^{\text {rd }}$ mode, this is not true. A comprehensive view of Figs. 7 through 14 shows that if we take into account the optimal damping effects in both the $1^{\text {st }}$ and $3^{\text {rd }}$ modes, it is best to place the DVA in the center of the beam with $m_{0}$ and $\widetilde{k}$ being approximately 0.1 and 0.2 , respectively. Clearly, the linear beam results (Eq. (50)) in Samani and Pellicano ${ }^{15}$ are not applicable to nonlinear beams. Furthermore, we considered the coupling of the $1^{\text {st }}$ and $3^{\text {rd }}$ modes in our model, which complicates the design of the DVA. Nevertheless, the optimal damping combination can still be identified using the 3DMACP. In the next section, we verify the accuracy of 3DMACPs using numerical methods.

\subsection{Analysis of Numerical Verification}

To conduct the numerical analysis of the beam equation including the DVA, we can use the mode shape in Eq. (21), substituting it into Eq. (7), and integrating it using the orthogonal properties to produce the following equations of motion to ex- 


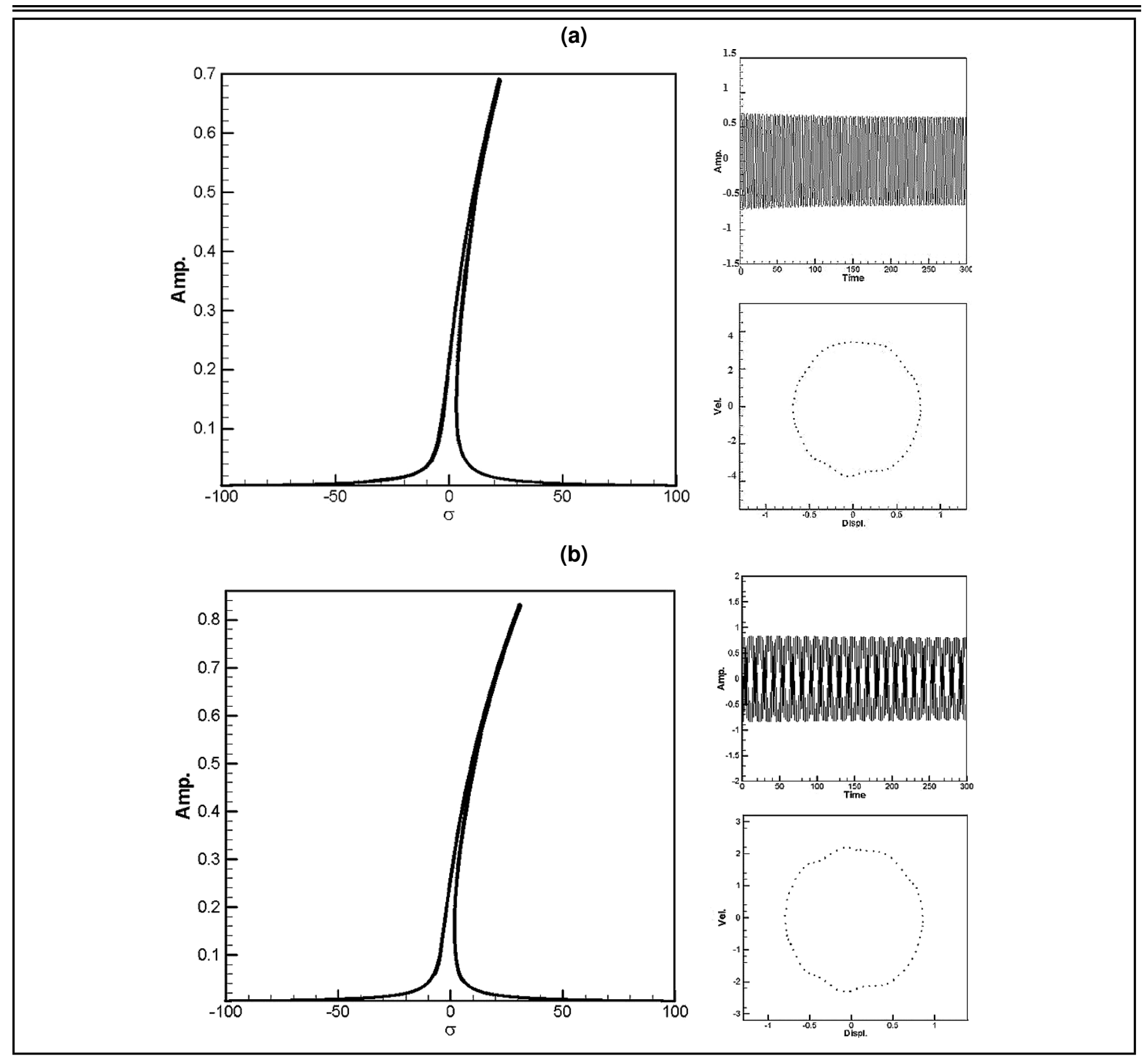

Figure 15. Numerical verification, (a) $m_{0}=0.15, \widetilde{k}=0.1, X_{D}=0.1$, (b) $m_{0}=0.02, \widetilde{k}=0.7, X_{D}=0.1$.

cite the $1^{\text {st }}$ mode:

$$
\begin{aligned}
& \stackrel{* *}{\xi_{1}}+\gamma_{1}^{4} \xi_{1}+\mu \stackrel{*}{\xi}_{1}+\bar{\omega}^{2} \xi_{1}+\frac{\hat{k}}{\int_{0}^{1} \phi_{1}^{2} d x} \Gamma_{1}+ \\
& \frac{1}{2} \gamma_{1}^{2} \xi_{1} \int_{0}^{1}\left(\gamma_{1}^{2} \xi_{1}^{2} \cos ^{2} \gamma_{1} x+\gamma_{3}^{2} \xi_{3}^{2} \cos ^{2} \gamma_{3} x\right) d x \\
& f_{s}\left[\xi_{1} \phi_{1}\left(X_{D}\right)+\xi_{3} \phi_{3}\left(X_{D}\right)-W_{D} \phi\left(X_{D}\right)\right]=\bar{F} \frac{\int_{0}^{1} \phi_{1} d x}{\int_{0}^{1} \phi_{1}^{2} d x}
\end{aligned}
$$

where

$$
\Gamma_{1}=\int_{0}^{1}\left(\xi_{1}^{3} \phi_{1}^{3}+3 \xi_{1}^{2} \phi_{1}^{2} \xi_{3} \phi_{3}+3 \xi_{1} \phi_{1} \xi_{3}^{2} \phi_{3}^{2}+\xi_{3}^{3} \phi_{3}^{3}\right) \phi_{1} d x
$$

and to excite the $3^{\text {rd }}$ mode:

$$
\begin{aligned}
& {\stackrel{* *}{\xi_{3}}}_{3}+\gamma_{3}^{4} \xi_{3}+\mu{\stackrel{*}{\xi_{3}}}_{3}+\bar{\omega}^{2} \xi_{3}+\frac{\hat{k}}{\int_{0}^{1} \phi_{3}^{2} d x} \Gamma_{3}+ \\
& \frac{1}{2} \gamma_{3}^{2} \xi_{3} \int_{0}^{1}\left(\gamma_{1}^{2} \xi_{1}^{2} \cos ^{2} \gamma_{1} x+\gamma_{3}^{2} \xi_{3}^{2} \cos ^{2} \gamma_{3} x\right) d x \\
& f_{s}\left[\xi_{1} \phi_{1}\left(X_{D}\right)+\xi_{3} \phi_{3}\left(X_{D}\right)-W_{D} \phi\left(X_{D}\right)\right]=\bar{F} \frac{\int_{0}^{1} \phi_{3} d x}{\int_{0}^{1} \phi_{3}^{2} d x} ;
\end{aligned}
$$

where

$$
\Gamma_{3}=\int_{0}^{1}\left(\xi_{1}^{3} \phi_{1}^{3}+3 \xi_{1}^{2} \phi_{1}^{2} \xi_{3} \phi_{3}+3 \xi_{1} \phi_{1} \xi_{3}^{2} \phi_{3}^{2}+\xi_{3}^{3} \phi_{3}^{3}\right) \phi_{3} d x
$$

Please refer to Appendix 2 for the relevant integration coefficients. We employed the RK4 approach to calculate displacements in the nonlinear beam with various parameter and location combinations when excited by a given external frequency. 


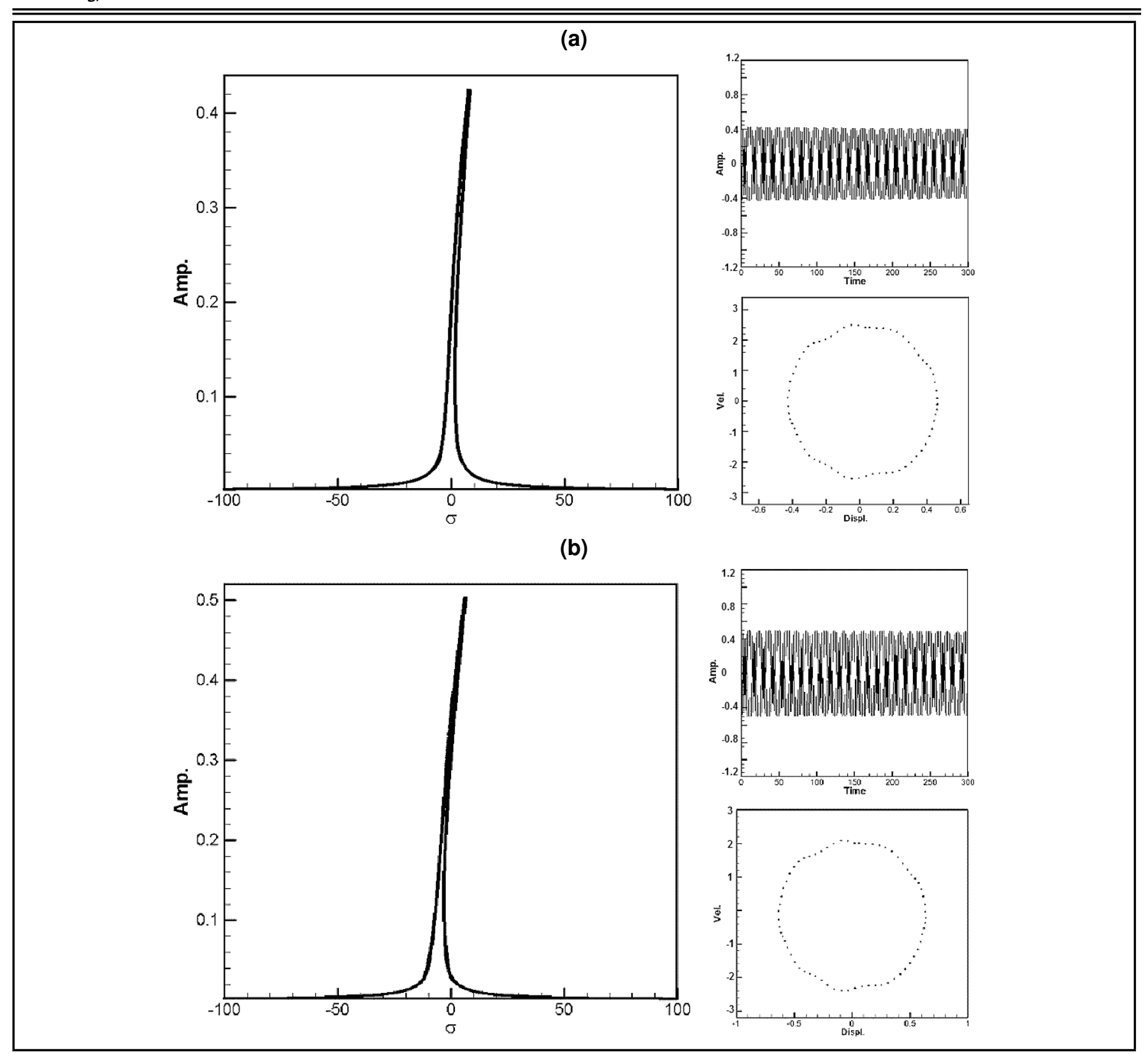

Figure 16. Numerical verification, (a) $m_{0}=0.15, \widetilde{k}=0.1, X_{D}=0.5$, (b) $m_{0}=0.02, \widetilde{k}=0.7, X_{D}=0.5$.

To verify the results from 3DMACPs (Figs. 7 to 14), we performed the numerical analysis and cross-referred the numerical results, fixed points plots and Poincaré maps (Figs. 15 to 18). The left part of Fig. 15a displays the fixed point plot of Fig. 7 with $m_{0}=0.15, \widetilde{k}=0.1$, and $X_{D}=0.1$ lain over the numerical results of the maximum amplitudes. The right upper graph of Fig. 15a displays the numerical result of time response and the lower graph is the numerical result of Poincaré map. Figure $15 \mathrm{~b}$ shows the same with $m_{0}=0.02, \widetilde{k}=0.7$, and $X_{D}=0.1$. The left part of Fig. 16a exhibits the fixed point plot of Fig. 10 with $m_{0}=0.15, \widetilde{k}=0.1$, and $X_{D}=0.5$ lain over the numerical results of the maximum amplitudes. The right upper graph of Fig. 16a displays the time response, and the lower graph is the Poincare map. Figure $16 \mathrm{~b}$ shows the same with $m_{0}=0.02, \widetilde{k}=0.7$, and $X_{D}=0.5$. As before, Fig. $17 \mathrm{a}$ displays the fixed point plot of Fig. 11 with $m_{0}=0.1, \widetilde{k}=0.1$, and $X_{D}=0.1$ lain over the numerical results of the maximum amplitudes, and Fig. 17b shows the same with $m_{0}=0.02$, $\widetilde{k}=0.7$, and $X_{D}=0.1$. Figure 18 a displays the fixed point plot of Fig. 14 with $m_{0}=0.1, \widetilde{k}=0.1$, and $X_{D}=0.5$ lain over the numerical results of the maximum amplitudes, and Fig. $18 \mathrm{~b}$ shows the same with $m_{0}=0.02, \widetilde{k}=0.4$, and $X_{D}=0.5$. Comparing the 3DMACPs with the fixed point plots and numerical method (time response), for each specified value of $m_{0}, \widetilde{k}$, and $X_{D}$, the maximum value of the fixed point plots agrees with the 3DMACP and the numerical results. We can see that the results are consistent, thereby demonstrating the accuracy of the model developed in this study and the concept of 3DMACPs.

\section{CONCLUSIONS}

In this study, we considered a hinged-hinged nonlinear beam supported or suspended by nonlinear springs to simulate a system that is suspended or placed on an elastic foundation. We subjected the main body to a harmonic force. Both ends of the nonlinear elastic beam are hinged; therefore, we had take into account the effects of stretching. We added a DVA to 


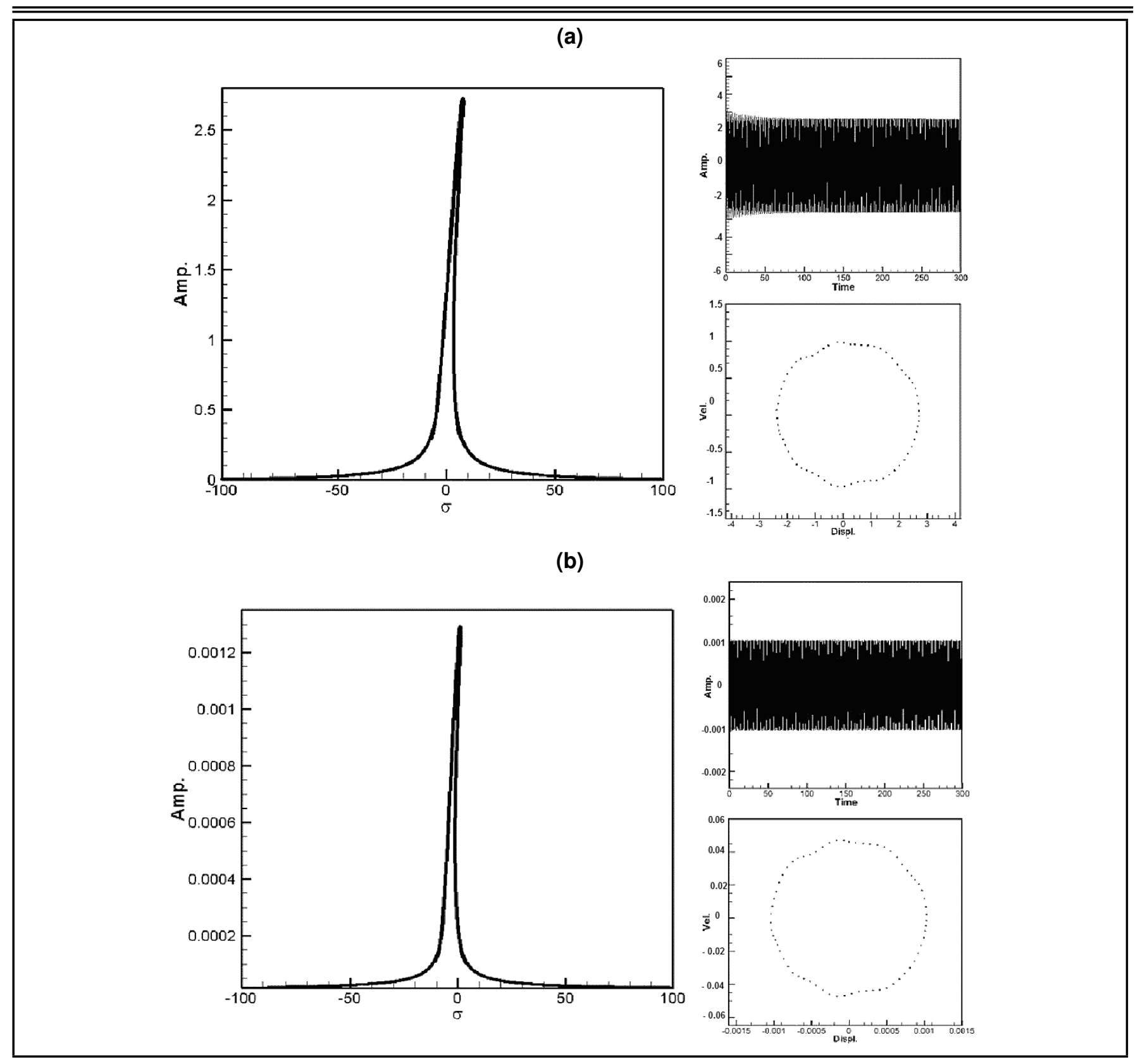

Figure 17. Numerical verification, (a) $m_{0}=0.1, \widetilde{k}=0.1, X_{D}=0.1$, (b) $m_{0}=0.02, \widetilde{k}=0.7, X_{D}=0.1$.

the beam system and investigated its effectiveness in damping when place in various locations. Using some elastic constant combinations, 1:3 internal resonance occurred in the $1^{\text {st }}$ and $3^{\text {rd }}$ modes. Placing the DVA at suitable locations was shown to prevent this, such that the damping effects of the DVA are more apparent in the $1^{\text {st }}$ mode than in the $3^{\text {rd }}$ mode. Damping performance was more significant when the DVA was closer to the center of the beam; however, 3DMACPs showed that optimal parameter combinations could also be found when the DVA was placed in other locations. For the $1^{\text {st }}$ mode, smaller elastic constants and larger mass ratios are not recommended. As for the $3^{\text {rd }}$ mode, damping performance is better when the DVA is near the center of the beam; however, the best results are obtained when the mass ratio is approximately 0.1 and the elastic constant is less than 0.2 . For the best damping performance in both the $1^{\text {st }}$ and $3^{\text {rd }}$ modes, the ideal combination includes the DVA at the center of the beam with the mass ratio and elastic constant of approximately 0.1 and 0.2 , respectively.

\section{ACKNOWLEDGEMENTS}

This research was supported by the Ministry of Science and Technology of Taiwan, Republic of China (grant number: MOST 103-2221-E-032-047).

\section{REFERENCES}

1 Nayfeh, A. H. and Mook, D. T. Nonlinear Oscillations, Wiley-Interscience, New York, (1979).

2 Nayfeh, A. H. and Balachandran, B. Applied Nonlinear Dynamics, Wiley-Interscience, New York, 158-172, (1995).

3 Nayfeh, A. H. and Pai, P. F. Linear and Nonlinear Structural Mechanics, New York, (2004).

$4 \mathrm{Ji}, \mathrm{Z}$. and $\mathrm{Zu}, \mathrm{J}$. W. Method of multiple scales for vibration analysis of rotor-shaft systems with non-linear bearing pedestal model, Journal of 


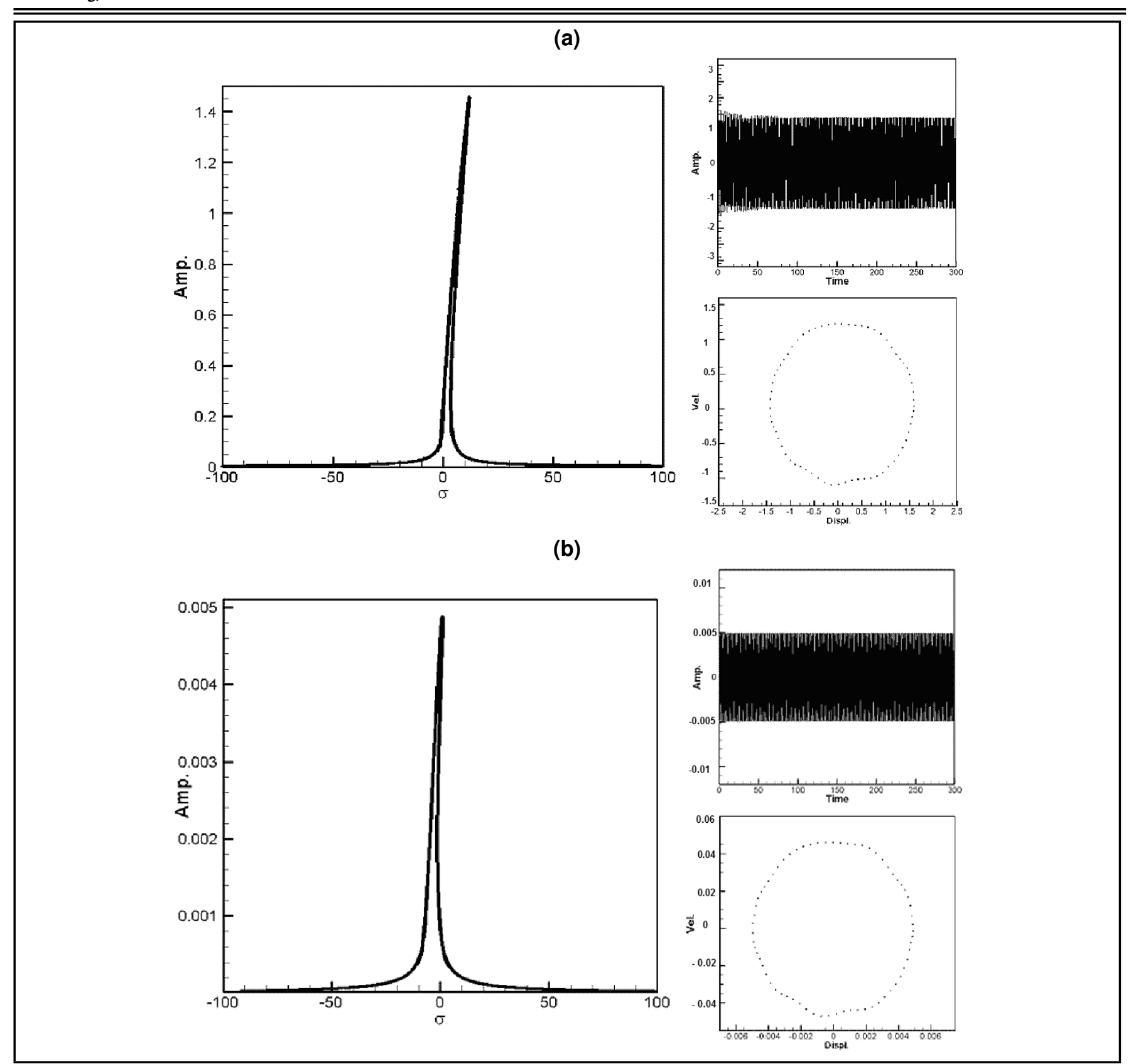

Figure 18. Numerical verification, (a) $m_{0}=0.1, \widetilde{k}=0.1, X_{D}=0.5$, (b) $m_{0}=0.02, \widetilde{k}=0.4, X_{D}=0.5$.

Sound and Vibration, 218 (2), 293-305, (1998). https://dx.doi.org/10.1006/jsvi.1998.1835

5 Nayfeh, A. H. and Nayfeh, S. A. On nonlinear modes of continuous systems, Transactions of the ASME, Journal of Vibration and Acoustics, 116 (1), 129-136, (1994). https://dx.doi.org/10.1115/1.2930388

6 Boertjens, G. J. and Van Horssen, W. T. On mode interactions for a weakly nonlinear beam equation, Nonlinear Dynamics, 17 (1), 23-40, (1998). https://dx.doi.org/10.1023/A:1008232515070

7 Mundrey, J. S. Railway Track Engineering, Tata McGrawHill, New Delhi, (2000).

${ }^{8} \mathrm{Fu}$, Y. M., Hong, J. W., and Wang, X. Q. Analysis of nonlinear vibration for embedded carbon nanotubes, Journal of Sound and Vibration, 296 (4-5), 746-756, (2006). https://dx.doi.org/10.1016/j.jsv.2006.02.024
9 Shen, H. S. A novel technique for nonlinear analysis of beams on two-parameter elastic foundations, International Journal of Structural Stability and Dynamics, 11 (6), 999-1014, (2011). https://dx.doi.org/10.1142/S0219455411004440

10 Wang, Y. R. and Chen, T. H. The vibration reduction analysis of a nonlinear rotating mechanism deck system, Journal of Mechanics, 24 (3), 253-266, (2008). https://dx.doi.org/10.1017/S1727719100002318

11 Wang, Y. R. and Chang, M. H. On the vibration reduction of a nonlinear support base with dual-shock-absorbers, Journal of Aeronautics, Astronautics and Aviation, Series A, 42 (3), 179-190, (2010). https://dx.doi.org/10.6125/JAAA

12 Wang, Y. R. and Chang, C. M. Elastic beam with nonlinear suspension and a dynamic vibration absorber at the free 
end, Transaction of the Canadian Society for Mechanical Engineering (TCSME), 38 (1), 107-137, (2014).

13 Nambu, Y., Yamamoto, S., and Chiba, M. A smart dynamic vibration absorber for suppressing the vibration of a string supported by flexible beams, Smart Materials and Structures, 23 (2), 025032, (2014). https://dx.doi.org/10.1088/0964-1726/23/2/025032

14 Tso, M. H., Yuan, J., and Wong, W. O. Suppression of random vibration in flexible structures using a hybrid vibration absorber, Journal of Sound and Vibration, 331 (5), 974986, (2012). https://dx.doi.org/10.1016/j.jsv.2011.10.017

15 Samani, F. S. and Pellicano, F. Vibration reduction of beams under successive traveling loads by means of linear and nonlinear dynamic absorbers, Journal of Sound and Vibration, 331 (10), 2272-2290, (2012). https://dx.doi.org/10.1016/j.jsv.2012.01.002

16 Wang, Y. R. and Lin, H. S. Stability analysis and vibration reduction for a two-dimensional nonlinear system, International Journal of Structural Stability and Dynamics, 13 (5), 1350031, (2013). https://dx.doi.org/10.1142/S0219455413500314

17 Wang, Y. R. and and Kuo, T. H. Effects of a dynamic vibration absorber on nonlinear hinged-free beam, ASCE Journal of Engineering Mechanics, 142 (4), (2016). https://dx.doi.org/10.1061/(ASCE)EM.19437889.0001039

18 Wang, Y. R. and Tu, S. C. Influence of tuned mass damper on fixed-free 3D nonlinear beam embedded in nonlinear elastic foundation, Meccanica, 51 (10), 2377-2416, (2016). https://dx.doi.org/10.1007/s11012-016-0372-8

19 Wang, Y. R. and Liang, T. W. Application of lumped-mass vibration absorber on the vibration reduction of a nonlinear beam-spring-mass system with internal resonances, Journal of Sound and Vibration, 350, 140-170, (2015). https://dx.doi.org/10.1016/j.jsv.2015.04.002

20 Ansari, R., Pourashraf, T., and Gholami, R. An exact solution for the nonlinear forced vibration of functionally graded nanobeams in thermal environment based on surface elasticity theory, Thin-Walled Structures, 93, 169-176, (2015). https://dx.doi.org/10.1016/j.tws.2015.03.013

21 Sayed, M. and Kamel, M. 1 to 2 and 1 to 3 internal resonance active absorber for non-linear vibrating system, $A p$ plied Mathematical Modelling, 36 (1), 310-332, (2012). https://dx.doi.org/10.1016/j.apm.2011.05.057

22 Ghayesh, M. H., Kazemirad, S., and Amabili, M. Coupled longitudinal-transverse dynamics of an axially moving beam with an internal resonance, Mechanism and Machine Theory, 52, 18-34, (2012). https://dx.doi.org/10.1016/j.mechmachtheory.2012.01.008

23 Ghayesh, M. H. and Amabili, M. Nonlinear dynamics of an axially moving Timoshenko beam with an internal resonance, Nonlinear Dynamics, 73 (1), 39-52, (2013). https://dx.doi.org/10.1007/s11071-013-0765-3
24 Ansari, R., Hasrati, E., Gholami, R., and, Sadeghi, F. Nonlinear analysis of forced vibration of nonlocal third-order shear deformable beam model of magneto-electro-thermo elastic nanobeams, Composites: $\quad$ Part B, 83, 226-241, (2015). https://dx.doi.org/10.1016/j.compositesb.2015.08.038

25 Ansari, R., Mohammadi, V., Faghih Shojaei, M., Gholami, R., and Sadeghi, F. Nonlinear forced vibration analysis of functionally graded carbon nanotube-reinforced composite Timoshenko beams, Composite Structures, 113, 316-327, (2014). https://dx.doi.org/10.1016/j.compstruct.2014.03.015

26 Ansari, R., Faghih Shojaei, M., Mohammadi, V., Gholami, R., and Sahmani, S. On the forced vibration analysis of Timoshenko nanobeams based on the surface stress elasticity theory, Composites: Part B, 60, 158-166, (2014). https://dx.doi.org/j.compositesb.2013.12.066

27 Ansari, R., Gholami, R., and Rouhi, H. Sizedependent nonlinear forced vibration analysis of magneto-electro-thermo-elastic Timoshenko nanobeams based upon the nonlocal elasticity theory, Composite Structures, 126, 216-226, (2015). https://dx.doi.org/10.1016/j.compstruct.2015.02.068

28 Ansari, R. and Gholami, R. Surface effect on the large amplitude periodic forced vibration of first-order shear deformable rectangular nanoplates with various edge supports, Acta Astronautica, 118, 72-89, (2016). https://dx.doi.org/10.1016/j.actaastro.2015.09.020

\section{APPENDIX 1}

$W=\frac{\bar{W}}{\bar{l}} ; \quad \tau=\frac{\bar{t}}{l^{2}}\left(\frac{E I_{A}}{\rho A}\right)^{1 / 2} ; \quad \omega=\left(\frac{E I_{A}}{\rho A \bar{l}^{4}}\right)^{1 / 2} ;$ $x=\frac{\bar{x}}{\bar{l}} ; \quad l=\frac{\bar{l}}{\bar{l}}=1 ; \quad \mu=\frac{\overline{m u} \bar{l}^{2}}{\left(\rho A E I_{A}\right)^{1 / 2}} ;$ $\hat{A}=\frac{E A r^{2}}{E I_{A}}-$ non-dimensional beam rigidity, and $r$ is the beam cross-section radius; $\quad \Omega=\overline{\Omega l}^{2}\left(\frac{\rho A}{E I_{A}}\right)^{1 / 2}$; $\bar{\omega}^{2}=\frac{k \bar{l}^{4}}{E I_{A}} ; \quad K=\frac{\beta r^{2} k \bar{l}^{4}}{E I_{A}} ; \quad m_{0}=\frac{\hat{m}}{\rho A \bar{l}} ;$ $\widetilde{k}=\frac{\bar{f}_{s}}{\omega^{2} \rho A \bar{l}} ; \quad \lambda=\frac{\bar{g}_{s}}{\omega \rho A \bar{l}}$.

\section{APPENDIX 2}

$$
\begin{aligned}
& \widetilde{k}_{m}=\frac{\widetilde{k}}{\int_{0}^{1} \sin ^{2} \gamma_{m} x d x} ; \quad A_{2 m}=\frac{\int_{0}^{1} \sin \gamma_{m} x d x}{\int_{0}^{1} \sin ^{2} \gamma_{m} x d x} \\
& A_{3 m}=\frac{1}{\int_{0}^{1} \sin ^{2} \gamma_{m} x d x}
\end{aligned}
$$

